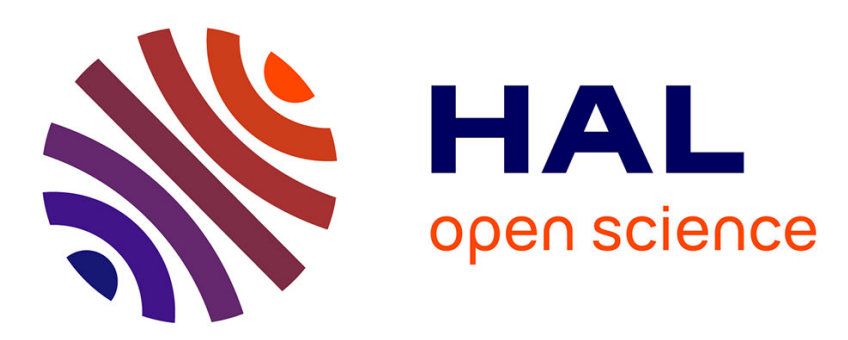

\title{
GeSi Raman spectra vs. local clustering/anticlustering: Percolation scheme and ab initio calculations
}

\author{
O. Pagès, R. Hajj Hussein, V. J. B. Torres
}

\section{To cite this version:}

O. Pagès, R. Hajj Hussein, V. J. B. Torres. GeSi Raman spectra vs. local clustering/anticlustering: Percolation scheme and ab initio calculations. Journal of Applied Physics, 2013, 114 (3), 10.1063/1.4813513 . hal-01517070

\section{HAL Id: hal-01517070 \\ https://hal.univ-lorraine.fr/hal-01517070}

Submitted on 11 May 2017

HAL is a multi-disciplinary open access archive for the deposit and dissemination of scientific research documents, whether they are published or not. The documents may come from teaching and research institutions in France or abroad, or from public or private research centers.
L'archive ouverte pluridisciplinaire HAL, est destinée au dépôt et à la diffusion de documents scientifiques de niveau recherche, publiés ou non, émanant des établissements d'enseignement et de recherche français ou étrangers, des laboratoires publics ou privés. 
GeSi Raman spectra vs. local clustering/anticlustering: Percolation scheme and ab initio calculations

O. Pagès' , R. Hajj Hussein, and V. J. B. Torres

Citation: Journal of Applied Physics 114, 033513 (2013); doi: 10.1063/1.4813513

View online: http://dx.doi.org/10.1063/1.4813513

View Table of Contents: http://aip.scitation.org/toc/jap/114/3

Published by the American Institute of Physics

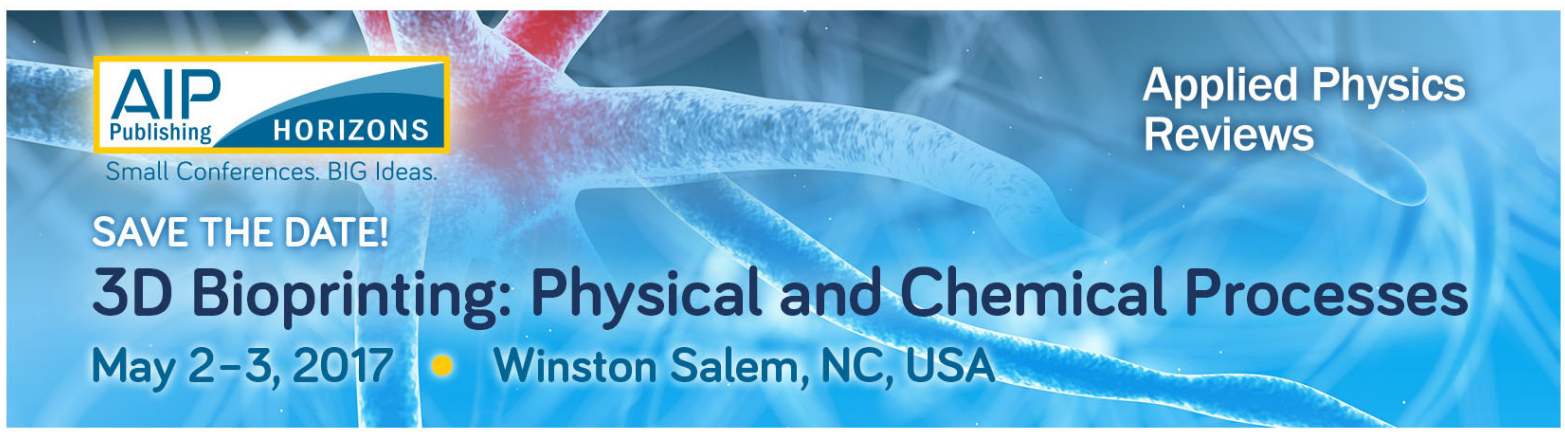




\title{
GeSi Raman spectra vs. local clustering/anticlustering: Percolation scheme and $a b$ initio calculations
}

\author{
O. Pagès, ${ }^{1, a)}$ R. Hajj Hussein, ${ }^{1}$ and V. J. B. Torres ${ }^{2}$ \\ ${ }^{1}$ Université de Lorraine, LCP-A2MC, Metz, 57078, France \\ ${ }^{2}$ Departamento de Física and I3N, Universidade de Aveiro, Campus Santiago, 3810-193 Aveiro, Portugal
}

(Received 19 April 2013; accepted 24 June 2013; published online 17 July 2013)

\begin{abstract}
We formalize within the percolation scheme that operates along the linear chain approximation, i.e., at one dimension (1D), an intrinsic ability behind Raman scattering to achieve a quantitative insight into local clustering/anticlustering in an alloy, using GeSi as a case study. For doing so, we derive general expressions of the individual fractions of the six GeSi percolation-type oscillators [ $1 \times(\mathrm{Ge}-\mathrm{Ge}), 3 \times(\mathrm{Ge}-\mathrm{Si}), 2 \times(\mathrm{Si}-\mathrm{Si})]$, which monitor directly the Raman intensities, via a relevant order parameter $\kappa$. This is introduced by adapting to the 1D oscillators of the GeSi-diamond version of the 1D-percolation scheme, i.e., along a fully consistent 1D treatment, the approach originally used by Verleur and Barker for the three-dimensional (3D) oscillators of their 1D-cluster scheme applying to zincblende alloys $[\mathrm{H}$. W. Verleur and A. S. Barker, Phys. Rev. 149, 715 (1966)], a somehow problematic one in fact, due to its 3D-1D ambivalence. Predictive $\kappa$-dependent intensity-interplays between the $\mathrm{Ge}_{0.5} \mathrm{Si}_{0.5}$ Raman lines are confronted with existing experimental data and with ab initio Raman spectra obtained by using (32-atom) disordered supercells matching the required $\kappa$ values, with special attention to the Ge-Si triplet and to the Si-Si doublet, respectively. @ 2013 AIP Publishing LLC. [http://dx.doi.org/10.1063/1.4813513]
\end{abstract}

\section{INTRODUCTION}

As soon as departing from pure (mono-constituent) media and considering mixtures, even involving only two substituent species, i.e., of the $\mathrm{A}_{1-\mathrm{y}} \mathrm{B}_{\mathrm{y}}$ type, one faces the key issue as how $\mathrm{A}$ and $\mathrm{B}$ arrange each other at a given composition $\mathrm{y}$. Is the A-to-B substitution random or is there any specific tendency for a given species (say A) to remain in its own local environment (A-like), or on the contrary to adopt a local environment mainly of the other (B) type, for some reason? Such deviations with respect to the ideal case of random Ato-B substitution are currently referred to as local clustering or local anticlustering, respectively. Semiconductor mixed crystals, of the zincblende type, like $\mathrm{Zn}_{1-\mathrm{y}} \mathrm{Be}_{\mathrm{y}} \mathrm{Se}$, or of the diamond type, with $\mathrm{Ge}_{\mathrm{y}} \mathrm{Si}_{1-\mathrm{y}}$ as leading system, can be considered as benchmark materials to address such issue. ${ }^{1}$ Indeed, they contain only two substituent species, each reducing to an atom (and not to a complicated molecule), moreover, disposed on a quasi regular and isotropic (cubic) lattice and attached to their immediate neighbors via strong (covalent) bonds.

In particular, the latter point concerning the chemical bonding is interesting with respect to local clustering/anticlustering (abbreviated c/ac hereafter). This is because the strength of a covalent bond, as currently measured at the laboratory scale via optical vibration (phonon) spectroscopies, such as Raman scattering or infrared (IR) absorption, is highly sensitive to its local environment. A naïve rule is that the bond force constant, and thus, the phonon frequency, ${ }^{2}$ falls down when a covalent bond is stretched, and vice versa.

\footnotetext{
a) Author to whom correspondence should be addressed. Electronic mail: olivier.pages@univ-lorraine.fr
}

For example, a spectacular Raman shift of $\sim 50 \mathrm{~cm}^{-1}$ is detected for the $\mathrm{Be}-\mathrm{Se}$ bond in $\mathrm{Zn}_{1-\mathrm{y}} \mathrm{Be}_{\mathrm{y}} \mathrm{Se}$, depending on whether a Be atom is isolated or paired to another $\mathrm{Be}$ atom (via an intermediary Se atom) in an otherwise pure $\mathrm{ZnSe}$ crystal $(x \sim 0){ }^{3}$ In contrast, the corresponding difference in Be-Se bond length, estimated around $2 \%$ from $a b$ initio calculations, ${ }^{3}$ could not be resolved in recent extended x-ray absorption fine structure (EXAFS) measurements on a synchrotron. ${ }^{4}$ This demonstrates that the bond force constant is potentially a more sensitive probe than the bond length to investigate the local environment of a bond. Apart from phonon spectroscopies (addressing the bond force constant) and EXAFS measurements (addressing the bond length), one may be tempted by $\mathrm{x}$-ray diffraction (addressing the lattice constant). ${ }^{1}$ However, the latter technique may be useful only when local c/ac leads to some periodical modulation of the alloy composition along certain crystal directions, corresponding to the formation of superlattice-like atom arrangements, at least on a restricted length scale. In this case, novel $\mathrm{x}$-ray lines, reflecting the periodicity of the superlattice, are expected on top of the primitive ones due to the crystal lattice itself. However, not all deviations from random substitution may lead to the emergence of such long range ordering. This might rather be the exception than the rule. Therefore, in most cases, x-ray diffraction might just be inappropriate. What remains then is our original suggestion to use the bond force constant. Thanks to its local character, it potentially constitutes a versatile probe, likely to address any type of local c/ac, in principle.

An interesting question is how the bond force constant can be used in practice to address local c/ac in an alloy? For simplicity, we start the discussion with $\mathrm{A}_{1-\mathrm{y}} \mathrm{B}_{\mathrm{y}} \mathrm{C}$ zincblende alloys, in which c/ac preserves the A-C (1-y) and B-C (y) 
bond fractions. This is because the A and B substituting species remain bonded to the same intercalated $\mathrm{C}$ atom in a zincblende alloy, whether the $\mathrm{A} \leftrightarrow \mathrm{B}$ substitution is random or not. In this case, one prerequisite to investigate local c/ac via Raman/IR spectroscopies is to have at one's disposal a distinct multi-mode signal per bond (explanation is given below). This makes sense in an alloy since the like bonds of a given species experience different distortions depending on their local environment, as needed to accommodate the local contrast in the bond physical properties (length, stiffness, ionicity, etc.) of the coexisting A-C and B-C species. This may lead to as many Raman/IR lines per bond (recall the naïve rule quoted in italics above), and hence to a 1-bond $\rightarrow$ multi-mode pattern per bond, in principle. From there, provided a one-dimensional (1D) approach of the lattice dynamics of an alloy can be adopted, corresponding to a description of the alloy along the linear chain approximation (LCA), then one disposes of a univocal correspondence between a given Raman line/frequency and a given "bond + environment" oscillator, to be discussed in terms of bond-stretching (1D). In this case, the individual fractions of such 1D-oscillators can be conveniently inferred from the intensities of the corresponding Raman lines, offering altogether, a detailed insight into the microstructure of an alloy. A basic conservation rule applies that the sum of the individual fractions of $1 \mathrm{D}$-oscillators due to a given bond matches the corresponding bond fraction, which remains invariant in a zincblende alloy, as already mentioned.

Somewhat paradoxically, such procedure becomes irrelevant when using a more realistic three-dimensional (3D) description of the lattice dynamics. This is because the univocal correspondence between a given Raman line and a given "bond + environment" motif, whereas valid at 1D, disappears at 3D, as extensively discussed in Refs. 5 and 6. Since they operate at the Brillouin zone center, optical phonon spectroscopies, such as Raman scattering and IR absorption provide a natural insight at $1 \mathrm{D}$ into the lattice dynamics of an alloy. Detail is given in Ref. 6. This has opened the way for three meaningful LCA-based (1D) models for the description of the Raman/IR of an alloy. Their main features are summarized below. We emphasize that Raman/IR spectroscopies detect only optic phonons at the Brillouin zone centre, corresponding to out-of-phase displacements of the intercalated $f c c$ sublattices in the zincblende or diamond structures, both taken as quasi rigid ones.

In fact, the LCA-based modified-random-element-isodisplacement (MREI: 1-bond $\rightarrow 1$-mode, 2-mode in total) model $^{7}$ and the LCA-based cluster (1-bond $\rightarrow 4$-mode, 8 modes in total) model, ${ }^{8}$ originally developed for zincblende alloys, and being used for decades to explain the Raman/IR spectra of such systems, either deny the existence of a 1-bond $\rightarrow$ multi-mode pattern (MREI model), which eliminates the sine qua non condition to access local c/ac via vibrational spectra, as explained above; or, when such multi-mode pattern is actually formalized (see below), the underlying approach is not free from conceptual ambiguity (cluster model), ${ }^{8,9}$ and thus, misleading in fine regarding the nature of the alloy disorder (in terms of random vs nonrandom). We may thus say that the presumed ability behind optical phonon spectroscopies to address local c/ac in a zincblende alloy remains unexploited in the traditional MREI and cluster schemes.

Over the past decade, an alternative LCA-based (1D) model has been developed, the so-called percolation model. This has lead to a unified understanding within a generic 1-bond $\rightarrow$ 2-mode (4-mode in total) behavior of the longstanding MREI/cluster-based classification of the Raman/IR spectra of $\mathrm{A}_{1-\mathrm{y}} \mathrm{B}_{\mathrm{y}} \mathrm{C}$ zincblende alloys including three main MREI sub-types (pure 2-mode, modified 2-mode, and 1-mode), plus a generic multi-mode type covered by the cluster scheme. ${ }^{5,10}$ In brief, the percolation model distinguishes between $\mathrm{AC}$ - and $\mathrm{BC}$-like environments for a given $\mathrm{A}-\mathrm{C}$ or B-C bond-stretching. What we retain is that this model combines the advantages of formalizing a proper multi-mode behavior per bond, as required to address local c/ac (see above)—in contrast with the MREI model, together with a consistent definition of the elementary oscillators at 1D, so as to remain in the spirit of the LCA, in contrast with the cluster model (in this model the elementary oscillators are defined at $3 \mathrm{D}$ as the possible first-neighbor environments of a given bond in the real crystal). ${ }^{5,6}$

It has already been shown how the percolation scheme can be used, in practice, to reveal a trend towards local clustering in the model $\mathrm{Zn}_{1-\mathrm{y}} \mathrm{Be}_{\mathrm{y}} \mathrm{Se}$ system. ${ }^{11}$ However, the discussion remained qualitative only. In particular, no order parameter could be introduced. This was due to an inability at the time to identify properly the microstructure of the percolation-type $\mathrm{AC}$ - and $\mathrm{BC}$-like 1D-environments of a bond, in terms of both length scale and composition.

Recently, a decisive forward step has allowed us to overcome such major drawback at the occasion of a successful generalization of the percolation scheme beyond zincblende alloys, to the leading diamond-type $\mathrm{Ge}_{\mathrm{y}} \mathrm{Si}_{1-\mathrm{y}}$ alloy. ${ }^{6}$ The percolation scheme for $\mathrm{Ge}_{\mathrm{x}} \mathrm{Si}_{1-\mathrm{y}}$ consists of the following six basic oscillators $\left[\mathrm{Ge}-\mathrm{Ge},(\mathrm{Ge}-\mathrm{Si})_{2}^{\mathrm{Ge}}\right.$, $\left.(\underline{G e}-S i){ }_{2}^{G e S i},(G e-S i)_{2}^{S i},(S i-S i)_{1}^{G e},(\underline{S i-S i})_{1}^{S i+G e S i}\right]$. In this notation, the main term refers to a given bond-stretching in a given 1D-environment whose length scale (first or second neighbors) and composition (Ge- or Si-like) are specified via a subscript $(1,2)$ and a superscript $(\mathrm{Ge}, \mathrm{Si})$, respectively. The underlined modes correspond to the so-called main $\mathrm{Ge}-\mathrm{Ge}, \mathrm{Ge}-\mathrm{Si}$, and $\mathrm{Si}-\mathrm{Si}$ modes in a crude 1-bond $\rightarrow 1$-mode MREI-like description of the GeSi Raman pattern. The alternative (not underlined) modes remain minor over most of the composition domain in the random $\mathrm{Ge}_{\mathrm{y}} \mathrm{Si}_{1-\mathrm{y}}$ alloy, ${ }^{6}$ and are currently referred to as the fine structure of the GeSi Raman spectra. We can be more explicit, for future use, by adding that the lower and upper Ge-Si oscillators are due to all-Ge (lower branch) and all-Si (upper branch) environments, while the remaining $(\mathrm{Ge}, \mathrm{Si})$-mixed environments give rise to a common oscillator in between (intermediary branch). We mention that in the random GeSi alloy the upper Ge-Si mode decomposes into a multiplet as soon as departing from the Si-parent limit, say beyond 30 at. \% Ge. The assignment of such multiplet is not clear yet, and a persisting source of problem when comparing experimental/ab initio $\mathrm{Ge}-\mathrm{Si}$ Raman data with calculated percolation-type Raman lineshapes, as done, e.g., in Sec. IV of the present work. The $\mathrm{Si}-\mathrm{Si}$ stretching, as for it, distinguishes between all-Ge 
(bottom branch) and alternative Si-like environments (top branch), i.e., including at least one $\mathrm{Si}$ atom. As for Ge-Ge, its stretching produces a unique Raman line at any alloy composition, reflecting a basic insensitivity of the Ge-Ge stretching to its local environment. Such detailed assignment of the percolation-type environments of a bond has allowed us to derive fully explicit fractions of the individual percolation-type 1D-oscillators depending on the alloy composition y for random $\mathrm{Ge}_{\mathrm{y}} \mathrm{Si}_{1-\mathrm{y}}$. Fig. 1 summarizes the content of the percolation scheme per oscillator for random $\mathrm{GeSi}$, regarding both the Raman-intensity, as monitored by the corresponding fraction of individual oscillator (general expressions explained in Sec. III A are reported in the body

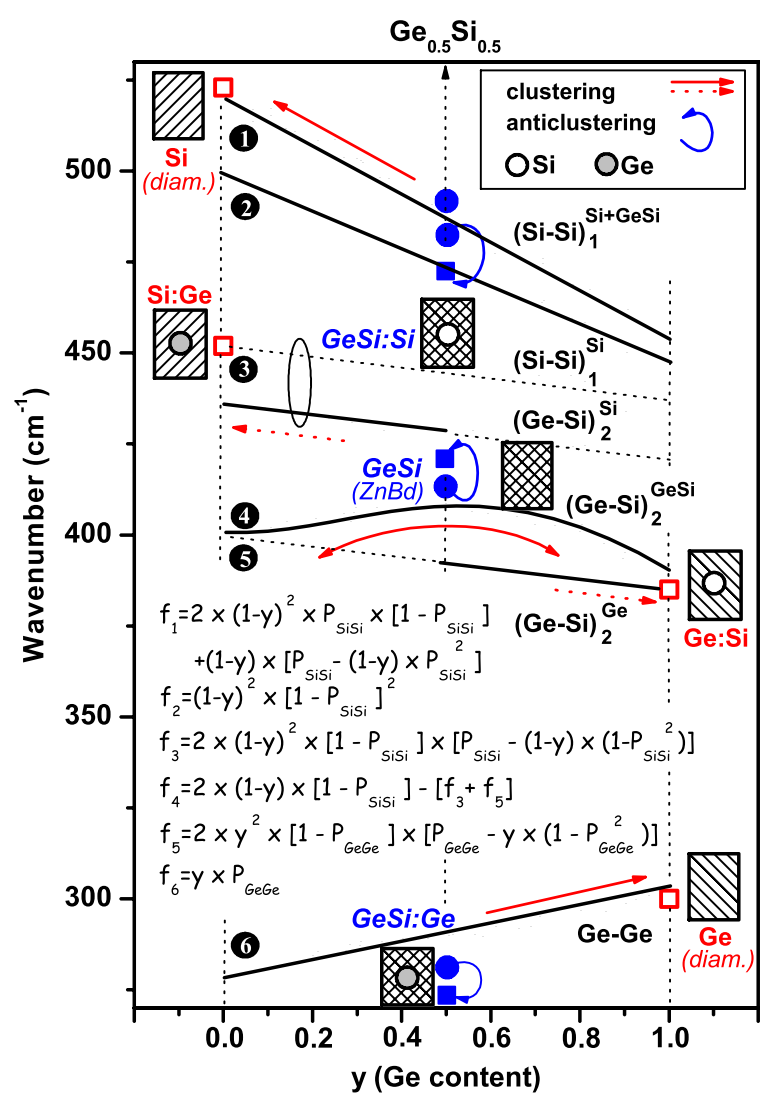

FIG. 1. Schematic $[1 \times(\mathrm{Ge}-\mathrm{Ge}), 3 \times(\mathrm{Ge}-\mathrm{Si}), 2 \times(\mathrm{Si}-\mathrm{Si})]$ percolation scheme for random $\mathrm{Ge}_{\mathrm{y}} \mathrm{Si}_{1-\mathrm{y}}$, as directly inspired from Ref. 6 (refer to Figs. 1 and 4 therein). On the right, the individual oscillators are labeled according to their standard percolation terminology, i.e., using a main term equipped with a superscript and a subscript, in reference to the considered bond-stretching and to the environment in which it takes place, with respect to both composition and length scale, respectively. A numerical labeling is also used (1-6), on the left, for more convenience, notably in a comparison with Figs. 3 and 5. Limit $a b$ initio frequencies calculated in diamond-Si (y $\sim 0$, oblique-left hatching) and diamond-Ge (y $\sim 1$, oblique-right hatching), on the one hand (open-red squares), and in zincblende-GeSi (y $\sim 0.5$, crossed hatching), on the other hand (plain-blue squares), using either pure supercells or containing a unique impurity (as schematically indicated), are used for a qualitative discussion of the frequency shifts of the main $\mathrm{Ge}_{0.5} \mathrm{Si}_{0.5}$ Raman features induced by clustering (red straight-curved arrows) or anticlustering (blue semi-closed loops/arrows). The ab initio frequencies of the unique $\mathrm{Ge}-\mathrm{Ge}$, main $\mathrm{Ge}-\mathrm{Si}$, and $\mathrm{Si}-\mathrm{Si}$ doublet in random- $\mathrm{Ge}_{0.5} \mathrm{Si}_{0.5}$, taken from the central curves $(\kappa=0)$ in Fig. 5, are added (plain-blue circles), for reference purpose. Globally, the same schematic code and labeling of the limit Raman frequencies is used in Fig. 5. The $\kappa$-dependence of the individual fractions of oscillators, which monitor directly the Raman intensities, is expressed via the $P_{S i S i}$ and $P_{G e G e}$ probabilities in the body of the figure. of the figure-see the $f_{i}$ terms, where $i$ refers to a given oscillator/branch as specified by black symbols), and the Ramanfrequency (plain lines). Extensive detail is available in Ref. 6.

Our ambition in this work is to achieve a versatile version of the percolation scheme for the lattice dynamics of an alloy, in which the individual fractions of oscillators, which monitor directly the Raman intensities, take a general form depending on a relevant parameter $\kappa$ of local c/ac, which remains to be defined. We emphasize that, by construction, the present approach is not relevant for the discussion of any c/ac-induced change in the Raman frequencies; only the Raman-intensity aspect is covered.

For working out such generalized $\kappa$-dependent version of the percolation scheme, we focus on the diamond-type $\mathrm{Ge}_{\mathrm{y}} \mathrm{Si}_{1-\mathrm{y}}$ alloy, and not on an $\mathrm{A}_{1-\mathrm{y}} \mathrm{B}_{\mathrm{y}} \mathrm{C}$ zincblende alloy. Indeed, we anticipate that the task may be more demanding, and thus, at the same time more general, for GeSi, corresponding to three bond species (Ge-Ge, Ge-Si, Si-Si), and six percolation oscillators in total, with bond environments defined up to second neighbors, ${ }^{6}$ than for any zincblende alloy, in which two bond species (A-C and B-C) offer only three percolation oscillators, moreover, restricted to the firstneighbor environment of a bond (see Refs. 5 and 10). Additional complication may arise in that, in a diamond alloy such as $\mathrm{Ge}_{\mathrm{y}} \mathrm{Si}_{1-\mathrm{y}}$, the $\mathrm{Ge}-\mathrm{Ge}, \mathrm{Ge}-\mathrm{Si}$, and $\mathrm{Si}-\mathrm{Si}$ bond fractions, which, respectively, scale as $y^{2}, 2 y \times(1-y)$ and $(1-y)^{2}$ in the random alloy, modify when c/ac develops. This is because any site of the diamond lattice is likely to be occupied by $\mathrm{Si}$ or $\mathrm{Ge}$ atoms, which thus may "see each other" as immediate neighbors, with concomitant impact on the $\mathrm{Si}-\mathrm{Si}, \mathrm{Ge}-\mathrm{Si}$, and $\mathrm{Ge}-\mathrm{Ge}$ bond fractions, and thus, also on the corresponding Raman intensities. For example, when assuming a random substitution at $\mathrm{y}=0.5$, the $\mathrm{Ge}-\mathrm{Ge}, \mathrm{Ge}-\mathrm{Si}$, and $\mathrm{Si}-\mathrm{Si}$ bond fractions amount to $0.25,0.5$, and 0.25 , respectively, and the $\mathrm{Ge}-\mathrm{Si}$ mixed-bond dominates. In case of full clustering, the Ge-Si bond just disappears-if we neglect the minor fraction at the frontier between the phaseseparated $\mathrm{Si}$ and $\mathrm{Ge}$ semi-infinite crystals. In contrast, local $\mathrm{c} / \mathrm{ac}$ preserves the $\mathrm{A}-\mathrm{C}$ and $\mathrm{B}-\mathrm{C}$ bond fractions in a $\mathrm{A}_{1-\mathrm{y}} \mathrm{B}_{\mathrm{y}} \mathrm{C}$ zincblende alloy, as already mentioned. In terms of oscillator strength, which physical parameter varies linearly with the fraction of oscillator, ${ }^{7}$ we may say that, in a diamond alloy such as GeSi, inter-bond transfer of oscillator strength, i.e., taking place in between Raman modes due to distinct bond species, superimposes onto the zincblende-like intra-bond transfer of oscillator strength driven by local c/ac, as merely concerned with the like Raman modes due to a given bond. In view of all this, the case of GeSi can be identified as a sort of bottleneck with respect to our ambition in this work to formalize the presumed sensitivity of optical phonon spectroscopies to local c/ac in an alloy. We are confident that, provided such generalized $\kappa$-dependent version of the percolation scheme can be worked out for the diamond-type GeSi alloy, then its transposition to any zincblende alloy should be straightforward.

In practice, the parameter $\kappa$ of local $\mathrm{c} / \mathrm{ac}$ is introduced in our GeSi percolation scheme following the approach earlier used by Verleur and Barker in their 1D cluster model that applies to the first neighbor $3 \mathrm{D}$-environments of a bond in a 
zincblende alloy (see above). ${ }^{8}$ In brief, such parameter $\kappa$ monitors the probability of finding a given atom besides another atom of the like species. Basically the probability is larger than the related fraction of atoms in the alloy in case of clustering $(\kappa>0)$, and smaller in case of anticlustering $(\kappa<0)$. Extensive detail is given in Sec. III A. Now, as already mentioned, the approach of Verleur and Barker is not free from conceptual ambiguity, due to its 1D (scalar equations of motion) vs. 3D (definition of elementary oscillators) ambivalence. In order to remove such ambiguity, we opt for a pure 1D approach, for sake of consistency with the LCA, upon which the percolation scheme relies. Moreover, the approach is presently generalized to a diamond alloy, i.e., $\mathrm{GeSi}$, and extended to second-neighbor environments besides first-neighbor ones, in reference to the $\mathrm{Ge}$-Si triplet and to the Si-Si doublet, respectively.

The manuscript is organized as follows. Based on selected experimental data in the literature, we identify in Sec. II the main features characterizing the dependence of the $\mathrm{GeSi}$ Raman spectra on c/ac, in view to test the predictions of the $\kappa$-dependent version of the $\mathrm{GeSi}$ percolation scheme. In Sec. III, we derive such generalized version of the GeSi percolation scheme, in which the individual fractions of 1D-oscillators are expressed via a proper parameter $\kappa$ of local c/ac. This is introduced along the approach used by Verleur and Barker in their 1D cluster model that applies to the first neighbor 3D-environments of a bond in a zincblende alloy. ${ }^{8}$ Only, in order to remove an ambiguity behind such 1D-3D ambivalence, we adopt a pure 1D formalism in this work, as detailed in Sec. III A. This applies to the diamond structure, using $\mathrm{Ge}_{y} \mathrm{Si}_{1-\mathrm{y}}$ as a case study, and, further, extends beyond the first-neighbor 1D-environment of a bond, up to the second-neighbor one, in reference to the $\mathrm{Si}-\mathrm{Si}$ doublet and to the $\mathrm{Ge}-\mathrm{Si}$ triplet, respectively. In $\mathrm{Sec}$. III B, predictive intensity-interplays between the $\mathrm{Ge}_{0.5} \mathrm{Si}_{0.5}$ Raman lines depending on clustering $(\kappa>0)$ and anticlustering $(\kappa<0)$ are produced both within the crude MREI-like scheme-thus, only concerned with the three main $\mathrm{Ge}-\mathrm{Ge}, \mathrm{Ge}-\mathrm{Si}$, and $\mathrm{Si}-\mathrm{Si}$ lines, for reference purpose, and also within the more refined percolation scheme-covering the main Raman features as well as the Ge-Si and $\mathrm{Si}-\mathrm{Si}$ fine structures, corresponding to six oscillators in total $[1 \times(\mathrm{Ge}-\mathrm{Ge}), 3 \times(\mathrm{Ge}-\mathrm{Si}), 2 \times(\mathrm{Si}-\mathrm{Si})]{ }^{6}$ The resulting $\kappa$-dependent percolation-type lineshapes are eventually confronted with part of the earlier selected experimental data (refer to Sec. II) on the one hand, in Sec. III C, mostly in reference to the Ge-Si triplet then, and with corresponding ab initio Raman spectra calculated with the AIMPRO code by using reasonably large (32-atom) fully relaxed supercells matching the required $\kappa$ values, on the other hand, in Sec. IV, searching then for a special insight into the $\mathrm{Si}-\mathrm{Si}$ doublet. A prerequisite for the discussion of the ab initio spectra is a proper assignment of the $\mathrm{Ge}-\mathrm{Ge}, \mathrm{Ge}-\mathrm{Si}$, and $\mathrm{Si}-\mathrm{Si}$ Raman spectral ranges depending on $\kappa$. This is achieved in Sec. IV A based on ab initio calculation of limit Raman frequencies arising from prototype impurity motifs in case of full c/ac. The effect of c/ac on the ab initio Raman intensities is then discussed in Sec. IVB, notably in its adequacy with the prediction of the percolation scheme. Last, a brief discussion of the $a b$ initio Raman frequencies in their $k$-dependence is also included, in Sec. IVC, for sake of completeness. Conclusions are summarized in Sec. V.

\section{GeSi RAMAN SPECTRA IN THEIR DEPENDENCE ON CLUSTERING/ANTICLUSTERING-MAIN FEATURES IN THE LITERATURE}

When searching for Raman data in the literature to be used as references in this work, we observe that basically all the existing experimental ${ }^{12-22}$ and theoretical ${ }^{20-25}$ studies of local c/ac so far reported with $\mathrm{Ge}_{\mathrm{y}} \mathrm{Si}_{1-\mathrm{y}}$ take place around $y=0.5$, within $10 \%$. Recently, the effect of c/ac was explored with alternative GeSi alloy compositions, ${ }^{26}$ but mostly in relation to the Raman frequencies, while our main concern in this work is the Raman intensities, as already explained. Interestingly, the three bond species are in comparable proportions in the random $\mathrm{Ge}_{0.5} \mathrm{Si}_{0.5}$ alloy (refer above). In fact, the three main $\mathrm{Ge}-\mathrm{Ge}, \mathrm{Ge}-\mathrm{Si}$, and $\mathrm{Si}-\mathrm{Si}$ Raman lines (underlined in the percolation series) exhibit similar intensities at $\mathrm{y} \sim 0.5$. Moreover, the three minor modes constituting the fine structure (not underlined), though comparatively weak, are clearly visible as well (for an overview see Fig. 5 of Ref. 6). At any other y value, at least one of the minor mode is missing. Therefore, $\mathrm{y} \sim 0.5$ is ideal when searching to detect any possible c/ac-induced deviation with respect to the nominal intensity balance between the $\mathrm{GeSi}$ Raman lines as observed in a random alloy. Additional interest arises in that $y=0.5$ is the only alloy composition at which both clustering and anticlustering can fully develop (detail is given in Sec. III B).

On the theoretical side, all types of atom arrangements can be generated in a supercell approach, in principle, provided the used supercell is large enough. This is not as simple experimentally. Intuitively, one may start to grow either a $(\mathrm{Ge})_{\mathrm{n}} /(\mathrm{Si})_{\mathrm{n}}$ superlattice, a short-period one so as to ensure a certain proximity of the $\mathrm{Si}$ and Ge atoms, or a zincblende GeSi compound, corresponding to limit cases of full clustering and full anticlustering, ${ }^{27}$ respectively. Then, progressive interdiffusion of $\mathrm{Si}$ and Ge might be finely tuned by applying some relevant cycle of thermal annealing until, at a certain stage, a random $(\mathrm{Ge}, \mathrm{Si})$ distribution is eventually achieved. We may already forget about the anticlustering $\rightarrow$ random route, simply because the starting zincblende GeSi crystal does not exist in reality. In contrast, high-quality short-period $\mathrm{Ge}_{n} \mathrm{Si}_{n}$ superlattices can be grown since the mid-eighties by using epitaxial techniques. ${ }^{13-22,26}$ This has offered a possibility to explore the alternative clustering $\rightarrow$ random route in several occasions, as summarized below.

We distinguish two different regimes along the clustering $\rightarrow$ random route, i.e., a "superlattice regime" in the early stages of the annealing process, in which a certain long range order is somehow preserved, and a "disordered regime" after intense annealing, in which the long range order is destroyed, resulting in a basically disordered alloy, not exclusive, though, of a reminiscent trend towards local clustering. The key point is that the two regimes correspond to different Raman patterns. The "superlattice regime," abundantly studied in the literature, both experimentally ${ }^{16-22}$ and theoretically, ${ }^{20-25}$ is characterized by specific Raman 
features, such as confined optical modes in the $\mathrm{Si}$ - or Ge-like layers, accompanied by acoustical modes resulting from zone folding due to the additional periodicity of the superlattice. ${ }^{18-22}$ Such complex vibration pattern falls beyond the scope of the percolation scheme and is not addressed in this work.

We focus on the "disordered regime," which starts as soon as the Raman pattern basically fits into the six-oscillator percolation scheme, and resembles that of a random $\mathrm{Ge}_{0.5} \mathrm{Si}_{0.5}$ alloy. Short-period superlattices with smeared, and not sharp, Ge/Si interfaces, reflecting significant $\mathrm{Ge} \leftrightarrow \mathrm{Si}$ intermixing, enter this category. We are aware of two detailed Raman studies on such systems, both concerned with $\mathrm{Ge}_{4} \mathrm{Si}_{4}$ superlattices. ${ }^{19,20}$ Shortperiod composition-modulated alloys-simply referred to as modulated alloys hereafter, corresponding to alternation of GeSi bi-layers with alloy compositions complementary to 0.5 into a superlattice sequence, such as those envisaged by Tsang et al. in their careful Raman study, ${ }^{15}$ are even better candidates for our use. This is because each bi-layer already consists of a disordered alloy (detail is given in Sec. IIIC), and not of a nominally pure $\mathrm{Si}$ or Ge layer as in the previous two examples of $\mathrm{Ge}_{4} \mathrm{Si}_{4}$ superlattices. Altogether, the experimental Raman spectra taken by Schorer et al., ${ }^{19}$ Alonso et al., ${ }^{20}$ and Tsang et al. ${ }^{15}$ constitute the corpus of $\mathrm{Ge}_{0.5} \mathrm{Si}_{0.5}$-related data that we will use to test the prediction of the generalized $\kappa$-dependent version of the GeSi percolation scheme. A selection of such Raman spectra is displayed in Fig. 2. The main trends observed with these systems are the following.

When clustering reduces, the main Ge-Si mode reinforces at the cost of the Ge-Ge and main Si-Si ones (underlined in the percolation series, see above), as expected within a crude MREI-like description. This simply reflects the reinforcement/disappearance of the corresponding bonds along the "clustering $\rightarrow$ random" route, respectively. From the magnitude of the deviation between the observed intensity ratios and the nominal ones for a random alloy, we may infer that the clustering is more pronounced for the $\mathrm{Ge}_{4} \mathrm{Si}_{4}$ superlattices (large deviation) than for the modulated alloy (small deviation). Nevertheless, in the latter case, the trend shows up unambiguously in the point-by-point difference spectrum carefully elaborated by Tsang et al. by subtracting to the Raman spectrum of their as-grown bilayer $\mathrm{Ge}_{0.5} \mathrm{Si}_{0.5}$ duperlattice, corresponding to moderate clustering, the one obtained with the same sample but after destruction of the long range order via intense annealing, thus, taken as nominally random. ${ }^{15}$ For a direct insight, the difference (top curve) and reference (bottom curve, shifted beneath) spectra in question are digitalized and reproduced in Fig. 2(a).

We want to be more accurate and turn now to the Ge-Si and $\mathrm{Si}-\mathrm{Si}$ fine structures (not underlined in the percolation series, see above), whose understanding falls beyond the MREI scheme. The dependence of such fine structure on c/ac has attracted little attention so far.

In the Ge-Si triplet, we observe that residual shoulders emerge on each side of the main/central Ge-Si mode in the difference Raman spectrum elaborated by Tsang et al. (refer to the vertical arrows in Fig. 2(a)). This indicates that the collapse of the latter mode with clustering (see above) goes with reinforcement of its two minor satellites.

As for the Si-Si doublet, careful examination of the difference Raman spectrum of Tsang et al. (refer to the top
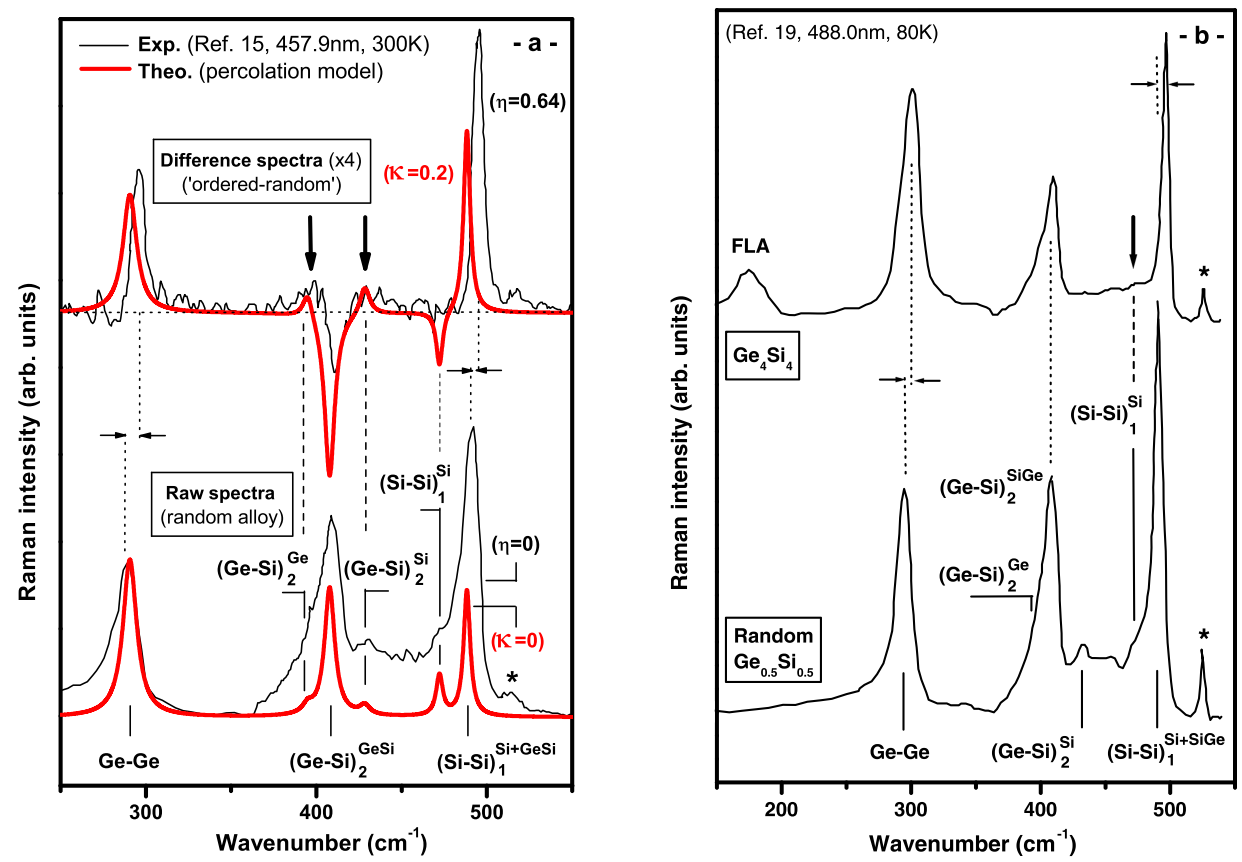

FIG. 2. Representative $\mathrm{Ge}_{0.5} \mathrm{Si}_{0.5}$ Raman spectra taken from the literature, used to reveal the effect of local clustering on the (a) Ge-Si (data digitalized from Fig. 1 of Ref. 15) and (b) Si-Si (data digitalized from Fig. 1 of Ref. 19) fine structures, as emphasized by vertical arrows in each panel. The spectra refer to epitaxial layers grown as random alloys (bottom curves in each panel) or under the form of superlattices (upper curves in each panel), corresponding either to a moderate clustering $\left[\eta=0.64\right.$, panel (a)] or to a $\mathrm{Ge}_{4} \mathrm{Si}_{4}$ sequence with interface mixing [panel (b)]. The stars refer to the underlying $\mathrm{Si}$ substrate in each case. In panel (a), the upper spectrum is the difference Raman spectrum obtained by subtracting the Raman spectra of the random alloy (bottom curve) from that of the superlattice $(\eta=0.64)$, multiplied by 4 (as indicated). Corresponding percolation-type Raman lineshapes (thick-red curves) for the random (bottom curve, $\kappa=0$ ) and clustered (upper curve, $\kappa=+0.2) \mathrm{Ge}_{0.5} \mathrm{Si}_{0.5}$ alloys are superimposed to the experimental data (thin-black ones), for comparison. The horizontal double-arrows mark significant phonon shifts with clustering. 
curve in Fig. 2(a)), does not reveal any significant change in the intensity of the lower/minor Si-Si satellite (refer to the thin dashed line at $470 \mathrm{~cm}^{-1}$ in Fig. 2(a)), while the upper/ main Si-Si mode strengthens (see above). The comparison between the Raman spectra of the highly ordered $\mathrm{Ge}_{4} \mathrm{Si}_{4}$ superlattices and of a reference random alloy (refer to the two bottom spectra in Fig. 1 of Ref. 18), as reproduced in Fig. 2(b), is more fruitful with this respect. It is striking that the minor $\mathrm{Si}-\mathrm{Si}$ feature, which shows up as a distinct shoulder besides the main Si-Si mode in the Raman spectrum of the reference random alloy (bottom curve), has totally disappeared with the $\mathrm{Ge}_{4} \mathrm{Si}_{4}$ clustered ones (upper curve, refer to the vertical arrow). This indicates that, under clustering, the reinforcement of the upper/main $\mathrm{Si}-\mathrm{Si}$ mode (see above) is accompanied by a progressive disappearance of its lower/ minor Si-Si satellite.

Summarizing, our selected data reveal the following intensity-interplays between the GeSi Raman lines when clustering develops. First, (i) the unique Ge-Ge and upper/ main $\mathrm{Si}-\mathrm{Si}$ features reinforce at the cost of the central/main Ge-Si one. This goes with (ii) reinforcement of the two satellite Ge-Si features, and (iii) progressive disappearance of the lower/minor $\mathrm{Si}-\mathrm{Si}$ satellite. By symmetry, we anticipate opposite trends in case of anticlustering, until in the final stage, corresponding to the formation of a pure zincblende GeSi crystal, i.e., the limit case of anticlustering, only the central/main Ge-Si mode survives. The $\kappa$-dependent version of the GeSi percolation scheme that we aim at deriving in this work should naturally incorporate features (i)-(ii)-(iii). This is a criterion for validation.

\section{GENERALIZED $\boldsymbol{\kappa}$-DEPENDENT VERSION OF THE GeSi PERCOLATION SCHEME}

$\mathrm{C} / \mathrm{ac}$ effects are taken into account in the diamond$\mathrm{GeSi}$ version of the percolation scheme by adapting to the corresponding 1D oscillators, as defined at the bond scale $(\mathrm{Ge}-\mathrm{Ge})$ or at the larger scale of the first ( $\mathrm{Si}-\mathrm{Si}$ ) and second (Ge-Si) neighbors of a bond, the approach originally developed by Verleur and Barker in their 1D cluster model for the 3D oscillators referring to all possible first neighbor environment of a bond in a zincblende alloy (four in total). ${ }^{8}$ A basic difference with their approach, though, is that we opt for a pure 1D formalism, for sake of consistency with the LCA upon which the percolation scheme relies. ${ }^{6}$

\section{A. Expression of the individual fractions of percolation-type GeSi 1D-oscillators in their dependence on $\boldsymbol{\kappa}$}

A relevant parameter $\kappa$ of local c/ac is introduced by materializing a trend towards $\mathrm{c} / \mathrm{ac}$ of a given substituting species in $\mathrm{Ge}_{\mathrm{y}} \mathrm{Si}_{1-\mathrm{y}}$, the trend being emphasized when the species gets more and more dilute. For example, we define $\mathrm{P}_{\mathrm{SiSi}}$ as the probability of finding one $\mathrm{Si}$ atom next to another $\mathrm{Si}$ atom on the $(\mathrm{Ge}, \mathrm{Si})$-diamond lattice at $1 \mathrm{D}$. In the random alloy, $\mathrm{P}_{\mathrm{SiSi}}$ is not dependent on the local neighborhood of an atom, thus, equal to the probability $\mathrm{P}_{\mathrm{Si}}$ of having one $\mathrm{Si}$ atom on a given site, which identifies with the fraction $(1-y)$ of $\mathrm{Si}$ atoms in the crystal. The trend towards local c/ac corresponds to an increased/decreased probability $\mathrm{P}_{\mathrm{SiSi}}$ with respect to the random case when the fraction of $\mathrm{Ge}$ atoms $(y)$ increases. Accordingly $\mathrm{P}_{\mathrm{SiSi}}$ may simply write as

$$
P_{S i S i}=(1-y)+\kappa_{1, S i} \times y,
$$

where the first subscript of $\kappa$ refers to the number of atoms added by the "starting" one to form the uniform (Si-like) 1D cluster under consideration (in this case, only one atom is added), and the second subscript refer to the cluster type ( $\mathrm{Si}$ - or Ge-like). Depending on the sign of $\kappa_{1, S i}$, this expression may as well be used to describe clustering $\left(\kappa_{1, S i}>0\right)$ or anti-clustering $\left(\kappa_{1, S i}<0\right.$, with some limitation then, as discussed below). A similar expression can be likewise defined for $P_{G e G e}$, corresponding to

$$
P_{G e G e}=y+\kappa_{1, G e} \times(1-y),
$$

with a similar notation. As a tendency towards c/ac of a given minor substituting species necessarily accompanies the same tendency for the other (dominant) substituting species, we may infer that $\kappa_{1, S i}$ and $\kappa_{1, G e}$ are related. The relation is expressed by equaling the two possible expressions of the probability of finding one $\mathrm{Si}$ atom next to one $\mathrm{Ge}$ atom in the crystal, with either Si or Ge as the "starting" site. This writes as

$$
P_{S i} \times P_{S i G e}=P_{G e} \times P_{G e S i}
$$

equivalent to

$$
(1-y) \times\left(1-P_{S i S i}\right)=y \times\left(1-P_{G e G e}\right),
$$

eventually leading to

$$
\kappa_{1, S i}=\kappa_{1, G e},
$$

abbreviated $\kappa_{1}=\kappa$ hereafter.

We may likewise define $P_{(\mathrm{SiSi} \ldots \mathrm{Si})_{n}}$ and $P_{(\mathrm{GeGe} \ldots \mathrm{Ge})_{n}}$ for 1D clusters constituted of $n$ like $\mathrm{Si}$ or Ge atoms, as

$$
\begin{gathered}
P_{(\mathrm{SiSi} \ldots \mathrm{Si})_{n}}=P_{S i}+\kappa_{n-1, S i} \times y, \\
P_{(\mathrm{GeGe} \ldots \mathrm{Ge})_{n}}=P_{G e}+\kappa_{n-1, G e} \times(1-y),
\end{gathered}
$$

with the same convention for the subscripts of $\kappa$. General forms of the $\kappa_{n-1, S i}$ values for $n$ equal to 2 and 3, which appears to be sufficient for our purpose, may then be inferred from the following series of correspondences, similar in nature to Eq. (3):

$$
P_{S i} \times P_{S i S i} \times P_{S i S i G e}=P_{G e} \times P_{G e S i} \times P_{G e S i S i} \quad\left(\text { access to } \kappa_{2, S i}\right),
$$

$$
\begin{aligned}
& P_{S i} \times P_{S i S i} \times P_{S i S i S i} \times P_{S i S i S i G e} \\
& \quad=P_{G e} \times P_{G e S i} \times P_{G e S i S i} \times P_{G e S i S i S i} \quad\left(\text { access to } \kappa_{3, S i}\right) .
\end{aligned}
$$

In expressing such correspondences we assume, like in Ref. 8, that 
$\forall X=(S i, G e)$ and $\forall n, P_{G e S i(X X \ldots X)_{n}}=P_{S i G e(X X \ldots X)_{n}}=P_{(X X \ldots X)_{n}}$

In fact, Eq. (7) reflects a technical inability behind our specific way to introduce $\kappa_{n, S i}$ to express any probability related to the formation of a given $1 \mathrm{D}$ cluster "starting" by a nonuniform succession of atoms. For example, $P_{\text {GeSiSi }}$ $=1-P_{\text {GeSiGe }}$ cannot be directly expressed nor via $P_{S i S i S i}$ neither via $P_{G e G e G e}$, but advantageously simplifies to $P_{S i}$ $=1-y$ when applying (7), in consideration of what $P_{S i S i G e}=1-P_{S i S i S i}$ may be derived, giving access to $\kappa_{2, S i}$. Symmetrical equations to Eqs. (6), in which Si is replaced by $\mathrm{Ge}$ and vice versa, are correspondingly used to access the $\kappa_{n, G e}$ values.

Using Eqs. (6) and (7), the following series of important probabilities may then be expressed via $P_{S i S i}$ and $P_{G e G e}$, whose dependencies on the order parameter $\kappa$ are specified according to Eqs. (1)

$$
\begin{aligned}
P_{S i S i S i}= & {\left[P_{S i S i}-(1-y) \times\left(1-P_{S i S i}\right)\right] \times P_{S i S i}^{-1}, } \\
P_{S i S i S i S i}= & {\left[P_{S i S i}-(1-y) \times\left(1-P_{S i S i}^{2}\right)\right] } \\
& \times\left[P_{S i S i}-(1-y) \times\left(1-P_{S i S i}\right)\right]^{-1},
\end{aligned}
$$

with similar expressions for $P_{G e G e G e}$ and $P_{G e G e G e G e}$ as obtained just by substituting $\mathrm{Ge}$ for $\mathrm{Si}$ in each subscript of Eq. (8). Such probabilities are the basic ingredients in the fractions of the individual 1D-oscillators of the GeSi percolation scheme. For example, the fraction of $\mathrm{Si}-\mathrm{Si}$ 1D-oscillator corresponding to $\mathrm{Si}-\mathrm{Si}$ stretching in a $(\mathrm{Ge}, \mathrm{Si})$ mixed first-neighbor environment, labeled $(\mathrm{Si}-\mathrm{Si})_{1}^{\mathrm{GeSi}}$ in the terminology of the percolation scheme, expresses according to $f\left\{(\mathrm{Si}-\mathrm{Si})_{1}^{\mathrm{GeSi}}\right\}=2 P_{S i} \times P_{S i S i} \times P_{S i S i S i}$ $\times P_{\text {SiSiSiGe }}$, where 2 refers to the number of possible orientations of the 1D-cluster under consideration, and $P_{\text {SiSiSiGe }}=1-P_{\text {SiSiSiSi }}$. We recall that the latter oscillator contributes, together with $(S i-S i)_{1}^{S i}$ that refers to $\mathrm{Si}-\mathrm{Si}$ stretching in a pure-Si first-neighbor environment, to the upper branch of the ( $\mathrm{Si}-\mathrm{Si}$ ) doublet in the $\mathrm{GeSi}$ percolation scheme. Finally, we obtain the following $\kappa$-dependent expressions for the individual fractions of the six 1Doscillators of the GeSi percolation scheme, hereafter ranked in order of increasing frequency

$$
\begin{gathered}
f_{6}=f\{(G e-G e)\}=y \times P_{G e G e}, \\
f_{5}=f\left\{(G e-S i)_{2}^{G e}\right\} \\
=2 y^{2} \times\left(1-P_{G e G e}\right) \times\left[P_{G e G e}-y \times\left(1-P_{G e G e}^{2}\right)\right], \\
f_{4}=f\left\{(G e-S i)_{2}^{G e S i}\right\} \\
=2(1-y) \times\left(1-P_{S i S i}\right) \\
-\left[f\left\{(S i-G e)_{2}^{G e}\right\}+f\left\{(S i-G e)_{2}^{S i}\right\}\right] \\
f_{3}=f\left\{(G e-S i)_{2}^{S i}\right\} \\
=2(1-y)^{2} \times\left(1-P P_{S i S i}\right) \\
\times\left[P_{S i S i}-(1-y) \times\left(1-P_{S i S i}^{2}\right)\right],
\end{gathered}
$$

$$
\begin{aligned}
& f_{2}=f\left\{(S i-S i)_{1}^{G e}\right\}=(1-y)^{2} \times\left(1-P_{S i S i}\right)^{2} \\
f_{2}= & f\left\{(S i-S i)_{1}^{G e S i+S i}\right\}=2(1-y)^{2} \times P_{S i S i} \times\left(1-P_{S i S i}\right) \\
+ & (1-y) \times\left[P_{S i S i}-(1-y) \times P_{S i S i}^{2}\right] .
\end{aligned}
$$

Based on such refined six-oscillator $[1 \times(\mathrm{Ge}-\mathrm{Ge})$, $3 \times(\mathrm{Ge}-\mathrm{Si}), 2 \times(\mathrm{Si}-\mathrm{Si})]$ percolation-type description, one may easily infer a more crude three-oscillator $[1 \times(\mathrm{Ge}-\mathrm{Ge})$, $1 \times(\mathrm{Ge}-\mathrm{Si}), 1 \times(\mathrm{Si}-\mathrm{Si})] \mathrm{MREI}-\mathrm{like}$ description. This comes to a vision in which the individual percolation-type three-mode and two-mode patterns of the Si-Ge and $\mathrm{Si}-\mathrm{Si}$ bonds, respectively, virtually condense into a unique feature per bond. The $\kappa$-dependent fractions of such presumed 1-bond $\rightarrow 1$-mode Ge-Ge, Ge-Si, and Si-Si MREI-like 1D-oscillators would accordingly identify with

$$
\begin{gathered}
f\{(G e-G e)\}=f_{6}, \\
f\{(G e-S i)\}=P_{S i} \times P_{S i G e}+P_{G e} \times P_{G e S i}=f_{5}+f_{4}+f_{3}, \\
f\{(S i-S i)\}=P_{S i} \times P_{S i S i}=f_{2}+f_{1} .
\end{gathered}
$$

Such expressions simply reflect basic conservation rules as concerned with the total fractions of $\mathrm{Ge}-\mathrm{Si}$ and $\mathrm{Si}-\mathrm{Si}$ bonds in the alloy for a given value of the order parameter $\kappa$. Note that in contrast with the A-C (1-y) and B-C (y) bond fractions in a $\mathrm{A}_{1-\mathrm{y}} \mathrm{B}_{\mathrm{y}} \mathrm{C}$ zincblende alloy, that remain stable under c/ac (as explained in Sec. I), the Ge-Ge, Ge-Si, and $\mathrm{Si}-\mathrm{Si}$ bond fractions of the $\mathrm{Ge}_{\mathrm{y}} \mathrm{Si}_{1-\mathrm{y}}$ diamond alloy, defined via Eqs. (10) are all $\kappa$-dependent. Such equations incorporate both intra-bond and inter-bond transfers of oscillator strength in the diamond GeSi alloy, while only intra-bond transfer of oscillator strength exists in a zincblende alloy.

\section{B. Percolation/MREI-like $\kappa$-dependent $\mathrm{Ge}_{0.5} \mathrm{Si}_{0.5}$ Raman lineshapes}

$\kappa$-dependent Raman lineshapes for the representative $\mathrm{Ge}_{0.5} \mathrm{Si}_{0.5}$ alloy corresponding to intermediary composition are calculated along the exact procedure detailed in Ref. 6, using the same input parameters, except that the individual fractions of oscillators $f_{i}$, which monitor the Raman intensities, are presently re-expressed by using the more general $\kappa$-dependent forms given in Eqs. (9). In particular, the individual Raman frequencies and linewidths are taken identical to the values found for the random $\mathrm{Ge}_{0.5} \mathrm{Si}_{0.5}$ alloy, simply because our formalism does not naturally incorporate any $\kappa$-dependence neither for the Raman frequency, as already discussed in Sec. I, nor for the phonon damping, which is sample-dependent. Both clustering $(0<\kappa<1)$ and anticlustering $(0>\kappa>-1)$ are considered besides the random case $(\kappa=0$, the corresponding fractions of oscillators are explicit, e.g., in Fig. 4 of Ref. 6). Two distinct sets of curves in Fig. 3 are displayed: one obtained within the six-oscillator GeSi percolation scheme (thick lines) using Eqs. (9), and one obtained within the more crude three-oscillator MREI-like scheme (thin lines) using Eqs. (10), added for comparison.

We emphasize that similar sets of curves can be derived at any alloy composition, in principle, not only at $\mathrm{y}=0.5$. 


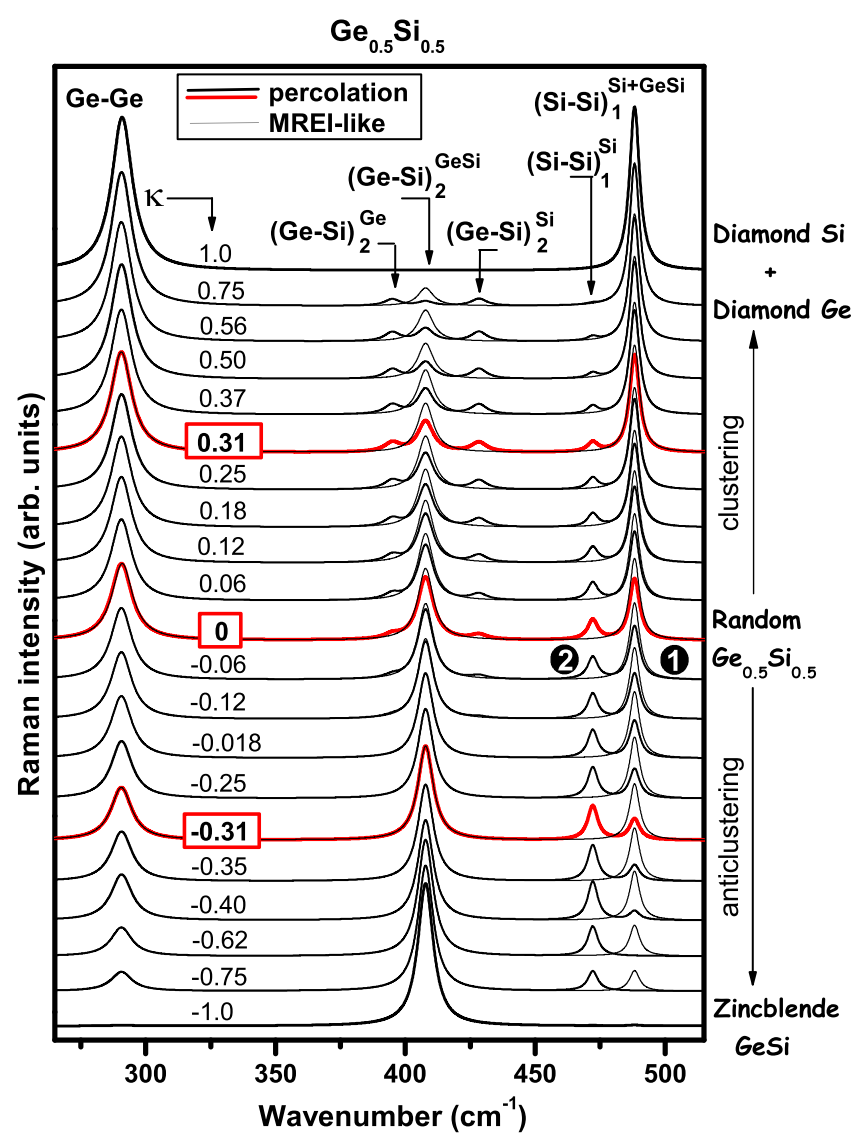

FIG. 3. $\kappa$-dependent percolation (black-thick curves) and MREI (black-thin curves) $\mathrm{Ge}_{0.5} \mathrm{Si}_{0.5}$ Raman lineshapes in case of clustering $(\kappa>0)$ and anticlustering $(\kappa<0)$, calculated by using the individual $\kappa$-dependent fractions of oscillators given in Fig. 1. The Raman frequencies and phonon damping are taken constant, identical to those in the random $\mathrm{Ge}_{0.5} \mathrm{Si}_{0.5}$ alloy, in a crude approximation. The percolation-type Raman spectra corresponding to the selected $\kappa$ values of $+0.31,0$, and -0.31 are emphasized (red curves). A direct comparison can be made with corresponding $\kappa$-dependent $a b$ initio Raman spectra reported in Fig. 5. Special attention may be awarded to the sensitive $\mathrm{Si}$-Si doublet identified by a specific labeling.

However, $\mathrm{Ge}_{0.5} \mathrm{Si}_{0.5}$ is particularly interesting in that the calculations can be pushed to the limit $\kappa$ values, corresponding to full clustering $(\kappa=+1)$ and full anticlustering $(\kappa=-1)$. At any other alloy composition, anticlustering cannot fully develop. This is because when departing from $y=0.5$ the major bond species has to remain segregated to a certain extent, at least locally. Technically, the anticlustering limit is achieved when the calculated fraction of the minor bond species becomes negative when using Eqs. (9) and (10), which is not realistic physically. Such situation is never encountered in case of clustering, which can fully develop at any alloy composition.

The basic trend with clustering is that the Ge-Ge and $\mathrm{Si}-\mathrm{Si}$ features reinforce at the cost of the intermediary $\mathrm{Ge}-\mathrm{Si}$ one, consistently with experimental findings [refer to (i) in Sec. II]. Ultimately $(\kappa=+1)$, only the Ge-Ge and $\mathrm{Si}-\mathrm{Si}$ signals survive. This reflects directly the abundance of the corresponding bonds in the crystal (see Sec. I). Careful examination of the fine structure in each spectral range further reveals that the Ge-Si side features reinforce at the cost of the central one (compare the MREI-thin and percolationthick Ge-Si lines), until at a certain stage $(\kappa \gtrsim 0.5)$ both side features become dominant. This conforms to intuition since the side and central modes refer to pure ( $\mathrm{Ge}$ or $\mathrm{Si}$ ) and (Ge,Si)-mixed environments (see Sec. I), thus, favored and disfavored by clustering, respectively. Again, this is consistent with experimental findings [refer to (ii) in Sec. II]. The trend is just opposite for the Si-Si doublet. The lower/minor $\mathrm{Si}-\mathrm{Si}$ satellite weakens with clustering, to the benefit of the upper/main Si-Si feature that reinforces (compare the MREIthin and percolation-thick $\mathrm{Si}$-Si lines). This makes perfect sense when realizing that the latter minor and dominant features are due to $\mathrm{Si}-\mathrm{Si}$ stretching in pure-Ge and Si-like environments, respectively (see Sec. I). The corresponding "bond + 1D-environments" oscillators, of the (Ge,Si)-mixed and Si-like types, are thus disfavored and favored by clustering, respectively. Again, this is consistent with experimental findings [refer to (iii) in Sec. II].

The $\kappa$-dependence of the multi-mode GeSi Raman intensities in case of anticlustering can be understood along the same lines, leading to reverse trends for the main as well as minor modes. We do not find it useful to enter more detail.

\section{Percolation vs. experimental insights-Main (Ge-Ge, Ge-Si, Si-Si) features and (Ge-Si) triplet}

In the following, we briefly test the $\kappa$-dependent version of the GeSi percolation scheme, in relation to points (i)-(iii) identified in Sec. II, on a quantitative basis. A natural experimental reference with this respect is the point-by-point difference Raman spectrum elaborated by Tsang et al. ${ }^{15}$ (refer to the upper curve in Fig. 2) by substracting the Raman spectrum from their modulated $\mathrm{Ge}_{0.5} \mathrm{Si}_{0.5}$ bilayer alloy, corresponding to moderate clustering, from that of a reference $\mathrm{Ge}_{0.5} \mathrm{Si}_{0.5}$ random alloy (corresponding to the bottom curve in Fig. 2(a)).

In view to elaborate a theoretical percolation-type difference Raman spectrum to compare with such experimental reference, we need to determine the relevant value of our vibrational-like order parameter $\kappa$, as defined within the percolation scheme, corresponding to the structural-like order parameter $\eta=0.64$, as independently determined by Tsang et al. for their modulated $\mathrm{Ge}_{0.5} \mathrm{Si}_{0.5}$ alloy by using $\mathrm{x}$-ray diffraction. ${ }^{15}$ In fact this alloy consists of a superlattice-like periodical modulation of the alloy composition along the [111] crystal axis under the form of a $G e_{0.5(1+\eta)} S i_{0.5(1-\eta)}$ $-G e_{0.5(1+\eta)} S i_{0.5(1-\eta)} / G e_{0.5(1-\eta)} S i_{0.5(1+\eta)}-G e_{0.5(1-\eta)} S i_{0.5(1+\eta)}$ bilayer alternation, in which closely (widely) spaced (111) planes are of the like (different) species. A simple bondcounting procedure leads to the following estimates of the individual bond fractions depending on $\eta$

$$
\begin{aligned}
f\{(S i-S i)\} \\
=\frac{1}{8}\left\{0.5(1+\eta) \times\left[0.5(1+\eta)+\sum_{u=0}^{3} u \times \frac{3 !}{u !(3-u) !}\right.\right. \\
\left.\left.\quad \times[0.5(1-\eta)]^{u} \times[0.5(1+\eta)]^{3-u}\right]+A\right\}
\end{aligned}
$$

being clear that

$$
f\{(G e-G e)\}=f\{(S i-S i)\},
$$


which implies

$$
f\{(G e-S i)\}=1-2 f\{(S i-S i)\},
$$

where $A$ represents a variant of the entire first term in Eq. (11a) in which $(1-\eta)$ is replaced by $(1+\eta)$, and vice versa. Accordingly, for $\eta=0.64$, the $\mathrm{Ge}-\mathrm{Ge}, \mathrm{Ge}-\mathrm{Si}$, and $\mathrm{Si}-\mathrm{Si}$ bond fractions amount to $\sim 30 \%, \sim 40 \%$, and $\sim 30 \%$, respectively, to compare with $25 \%, 50 \%$, and $25 \%$ in the random alloy $(\eta=0$, see Sec. I). A similar distribution of bond fractions is achieved via Eqs. (10) by taking $\kappa \sim 0.20$.

We expect that the theoretical "clustered-random" difference spectrum obtained by subtracting the percolationtype Raman spectrum due to the $\mathrm{Ge}_{0.5} \mathrm{Si}_{0.5}$ random alloy, defined by $\kappa \sim 0$, from the corresponding spectrum due to the partially ordered alloy, characterized by $\kappa \sim 0.20$, more or less matches the experimental difference spectrum obtained by Tsang et al. For a direct comparison, we superimpose in Fig. 2(a) the theoretical percolation-type Raman lineshapes obtained for the difference spectrum (top curve), and also for the reference random alloy (bottom curve), onto the corresponding experimental data of Tsang et al. In the latter case, the superimposition is done after normalization to the intensity of the unique Ge-Ge mode. Note that the theoretical percolation-type Raman lineshape for random $\mathrm{Ge}_{0.5} \mathrm{Si}_{0.5}$ is similar in every respect (phonon dampings, Raman frequencies, and Raman intensities) to the corresponding generic curve earlier derived in Ref. 6 (refer to $\mathrm{y}=0.5$ in Fig. 5 therein).

First, we compare briefly the experimental and theoretical Raman spectra of the reference random alloy (refer to the bottom curves in Fig. 2(a)). We are forced to admit that there is a problem with the intensities of the main $\mathrm{Ge}-\mathrm{Si}$ and $\mathrm{Si}-\mathrm{Si}$ modes, the latter showing up much more strongly in the experimental spectrum than in the theoretical one. In fact, this must not be so surprising since Tsang et al. did record their reference Raman spectrum under near resonant condition, i.e., by using the $457.9 \mathrm{~nm}$ excitation at $300 \mathrm{~K}$, leading to significant enhancement of the $\mathrm{Si}-\mathrm{Si}$ and Ge-Si Raman signals (refer to Figs. 2 and 3 of Ref. 19) with respect to the $\mathrm{Ge}-\mathrm{Ge}$ one, that remains basically insensitive to this particular resonance (Ref. 19). Obviously, such resonance effects were not incorporated into the percolation scheme. We recall that the intrinsic Raman efficiencies of the Ge-Ge, Ge-Si, and $\mathrm{Si}$-Si bonds were directly calibrated by implementing $a b$ initio calculations in pure diamond-Si, zincblende-GeSi, and diamond-Ge supercells out-of-resonance condition, respectively. ${ }^{6}$ In fact, the "starting discrepancy" between experience and theory concerning the Raman spectrum of the random alloy is not so dramatic when realizing that our main purpose is to compare the experimental and theoretical "clustered-random" difference Raman spectra (in reference to the top curves in Fig. 2(a)). In principle, the "starting discrepancy" should be suppressed in the difference spectrum.

In fact, the experimental and theoretical "clusteredrandom" difference spectra appear to be remarkably consistent in view of the simplicity of our percolation approach, especially in what concerns the intensities of the main features and of the Ge-Si triplet. The basic experimental trend, i.e., a reinforcement of the unique $\mathrm{Ge}-\mathrm{Ge}$ and upper/main $\mathrm{Si}$ $\mathrm{Si}$ modes, accompanied by a collapse of the main/central Ge-Si mode, is well-reproduced in the percolation-like theoretical curve. More interestingly, the two small resonances that emerge on each side of the main $\mathrm{Si}-\mathrm{Ge}$ antiresonance in the experimental difference show up clearly in the percolation-type difference. The situation is not as clear with the $\mathrm{Si}$-Si doublet. Indeed, the theoretical antiresonance due to the lower/minor $\mathrm{Si}-\mathrm{Si}$ mode does not show up in the experimental curve. Our present view is that the $\kappa$-difference between the modulated alloy $(\kappa \sim 0.2)$ and the random one $(\kappa \sim 0)$, is not sufficiently large to generate unambiguous impact on the Raman intensity of the latter mode, a rather broad/overdamped one in fact. Additional complication arises in that the main Si-Si Raman signal exhibits a significant blue-shift with clustering (see the double horizontal arrow in Fig. 2(a)), which cannot be accounted for within our percolation approach, as already explained. While the shift in question is rather small, i.e., of the order of $5 \mathrm{~cm}^{-1}$, it is not negligible for all that, representing approximately onethird of the Si-Si percolation splitting. In the experimental difference spectrum, such shift may lead to an artificial screening of the antiresonance due to the minor Si-Si component, as predicted within the percolation scheme when assuming a strict invariance of the Raman frequencies.

For unambiguous insight into the particular $\kappa$-dependence of the $\mathrm{Si}-\mathrm{Si}$ doublet, we may thus need to consider a larger $\kappa$-difference. Due to the lack of experimental data, we opt for a direct $a b$ initio insight.

\section{PERCOLATION VS. AB INITIO INSIGHTS-(Si-Si) DOUBLET}

Ab initio GeSi Raman spectra are calculated by using a pseudopotential spin density-functional supercell code (AIMPRO), ${ }^{28,29}$ along the local exchange-correlation parametrization by Perdew and Wang, ${ }^{30}$ taking the potentials for $\mathrm{Si}$ and $\mathrm{Ge}$ as proposed by Hartwigsen et al. (Ref. 31). The wave function was expanded in Cartesian Gaussian Atomic Orbitals (CGO). A contracted CGOs basis of $s, p$ and $d$-type was centered on each $\mathrm{Ge}$ or $\mathrm{Si}$ atom, in a total of 13 functions per atom. More details may be found in Ref. 32. Basically, we follow the same procedure earlier adopted to optimize GeSi supercells (Ref. 33), but generally using a thinner $12 \times 12 \times 6 k$-point mesh as proposed by Monkhorst and Pack (Ref. 34), for the integration on Brillouin zone. This k-sampling allows full convergence on the lattice parameters (a), bulk moduli (B), pressure derivatives of bulk modulus $\left(\mathrm{B}^{\prime}\right)$, and Raman frequencies $\left(\omega_{R}\right)$ of bulk Si, bulk $\mathrm{Ge}$ and bulk zincblende GeSi-which constitute natural references, as shown in Table I, and achieves very good agreement when compared with the experimental data (see Table I). Note that a $12 \times 12 \times 6 k$-point mesh on the 32 -atom supercell produces the same results as a $\mathrm{k}$-sampling converged cubic 64-atom supercell (see Table I), meaning also a convergence on supercell size.

The ab initio Raman spectra are obtained mostly by using 32-atom tetragonal GeSi supercells, after full relaxation (atom positions, lattice parameter, shape). The AIMPRO 
TABLE I. Ab initio values of the lattice parameter (a), bulk modulus (B), pressure derivative of bulk modulus ( $\left.\mathrm{B}^{\prime}\right)$, and Raman frequency $\left(\omega_{\mathrm{R}}\right)$ of pure silicon, pure germanium, and zincblende $\mathrm{GeSi}$, as obtained with different supercell sizes (differentiated by the number of atoms). The k-sampling is specified in each case.

\begin{tabular}{|c|c|c|c|c|c|c|c|c|c|}
\hline \multirow[b]{2}{*}{ Supercell } & \multicolumn{3}{|c|}{$\mathrm{Si}$} & \multicolumn{3}{|c|}{$\mathrm{Ge}$} & \multicolumn{3}{|c|}{ zincblende SiGe } \\
\hline & 32 & 64 & Exp. & 32 & 64 & Exp. & 32 & 64 & Alternative Theo. \\
\hline k-sampling & $12 \times 12 \times 6$ & $6 \times 6 \times 6$ & & $12 \times 12 \times 6$ & $6 \times 6 \times 6$ & & $12 \times 12 \times 6$ & $6 \times 6 \times 6$ & \\
\hline $\mathrm{a}(\mathrm{nm})$ & 0.540 & 0.539 & $0.543^{\mathrm{a}}$ & 0.558 & 0.558 & $0.565^{\mathrm{b}}$ & 0.548 & 0.548 & $0.548^{\mathrm{c}}$ \\
\hline $\mathrm{B}(\mathrm{GPa})$ & 98.5 & 98.5 & $97.9^{\mathrm{a}}$ & 74.4 & 74.4 & $77.2^{\mathrm{b}}$ & 86.8 & 86.8 & $89.4^{\mathrm{c}}$ \\
\hline $\mathrm{B}^{\prime}$ & 4.2 & 4.2 & $4.24^{\mathrm{d}}$ & 4.7 & 4.7 & $4.55^{\mathrm{d}}$ & 4.5 & 4.4 & \\
\hline$\omega_{\mathrm{R}}\left(\mathrm{cm}^{-1}\right)$ & 525 & 525 & $522^{\mathrm{e}}$ & 302 & 302 & $301^{\mathrm{f}}$ & 422 & 422 & \\
\hline
\end{tabular}

${ }^{\mathrm{a}}$ Reference 35 .

${ }^{\mathrm{b}}$ Reference 38 .

${ }^{\mathrm{c}}$ Reference 40.

${ }^{\mathrm{d}}$ Reference 36.

${ }^{\mathrm{e}}$ Reference 37.

${ }^{\mathrm{f}}$ Reference 39

$\operatorname{code}^{28}$ uses downhill simplex optimization to minimize the total energy with respect to one or all of the independent lattice parameters (including angles), and a conjugate gradient method to relax the atomic positions. We have checked that the two independent lattice parameters of each parallelepiped supercell representing the above crystals differ by only $10^{-6}$ au (atomic units). When using disordered $\mathrm{Ge}_{0.5} \mathrm{Si}_{0.5}$ supercells with different amount of clustering, as specified below, the lattice relaxation was performed with and without shape restriction, for comparison. For each type of supercell, the difference in lattice parameter does not exceed $10^{-4}$ au (atomic units). Also, the orthogonal angles between the parallelepiped directions are preserved within less than $0.001^{\circ}$. Last, the minimum in total energy, corresponding to optimum supercell relaxation, remains stable within $10^{-5} \mathrm{eV}$. In view of all this, we may safely state that there is no shape effect. Cluster calculations, sometimes preferred, such as, e.g., in the recent work of Abduljalil et al. dedicated to $\mathrm{GeSi}$ nanoalloys, ${ }^{41}$ are thus not required in the present case. Basically, while the $\mathrm{Si}-\mathrm{Si}, \mathrm{Ge}-\mathrm{Ge}$, and $\mathrm{Ge}-\mathrm{Si}$ bond lengths are significantly different (see Table I), with concomitant impact on the volume and atom positions after relaxation, it seems that the lattice parameter is affected in the same way along the three orthogonal directions of our tetragonal 32-atom $\mathrm{Ge}_{0.5} \mathrm{Si}_{0.5}$ disordered supercells. This remains evidently valid for the three additional 64-atom disordered supercells that we consider, referring to one isolated $\mathrm{Si}$ or $\mathrm{Ge}$ impurity in otherwise pure diamond-Si, diamond-Ge or zincblende GeSi-supercells.

A representative $a b$ initio insight into the Raman spectra of disordered $\mathrm{Ge}_{0.5} \mathrm{Si}_{0.5}$ is obtained by using 32-atom $\mathrm{Ge}_{0.5} \mathrm{Si}_{0.5}$ supercells of three different types, corresponding to local clustering $(\kappa>0)$ and local anticlustering $(\kappa<0)$ besides random $\mathrm{Ge} \leftrightarrow \mathrm{Si}$ substitution $(\kappa=0)$. The finite $\kappa$ values are taken symmetrical and as large as possible while maintaining at the same time a minimum bond counting of ten per species (out of 64 bonds in total per supercell) for a reliable statistics, being clear that the statistics of the alloy disorder is anyway not fully taken into account due to the finite size of the supercells. A relevant set of $\kappa$ values matching the above criteria is $(+0.31,0,-0.31)$, corresponding to the (Ge-Ge, Ge-Si, Si-Si) bond counting per supercell of $(21,22,21),(16,32,16)$, and $(11,42,11)$, respectively, as governed by Eqs. (10). The Si (small-blue symbol) and $\mathrm{Ge}$ (large-yellow symbol) atom arrangements in the successive (111) planes of the supercells in question are shown in Fig. 4.

Interestingly, such $\kappa$-set covers three distinct types of intensity-interplays within the $\mathrm{Si}-\mathrm{Si}$ percolation doublet when $\kappa$ varies, in reference to Fig. 3. One in which the lower

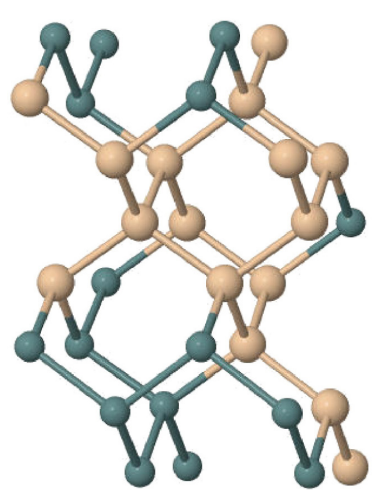

$\kappa=+0.31$

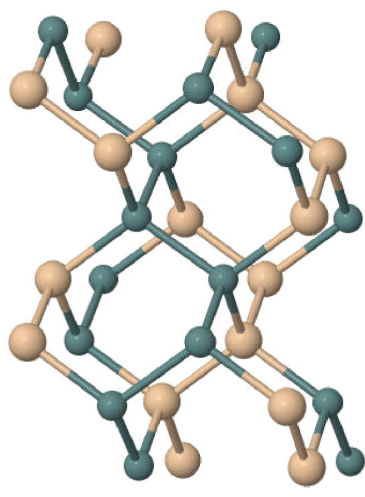

$\kappa=0$

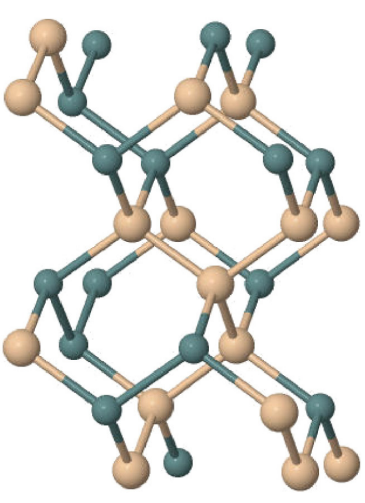

$\kappa=-0.31$
FIG. 4. Positioning of the Si (smallgreen symbol) and Ge (large-yellow symbol) atoms in three selected 32-atom $\mathrm{Ge}_{0.5} \mathrm{Si}_{0.5}$ supercells corresponding to clustering ( $\kappa=+0.31$, left position) random substitution $(\kappa=0$, center position), and anticlustering $(\kappa=-0.31$, right position). Identical $\kappa$ values are obtained just by inverting the $\mathrm{Si}$ and $\mathrm{Ge}$ atoms in each supercell. 


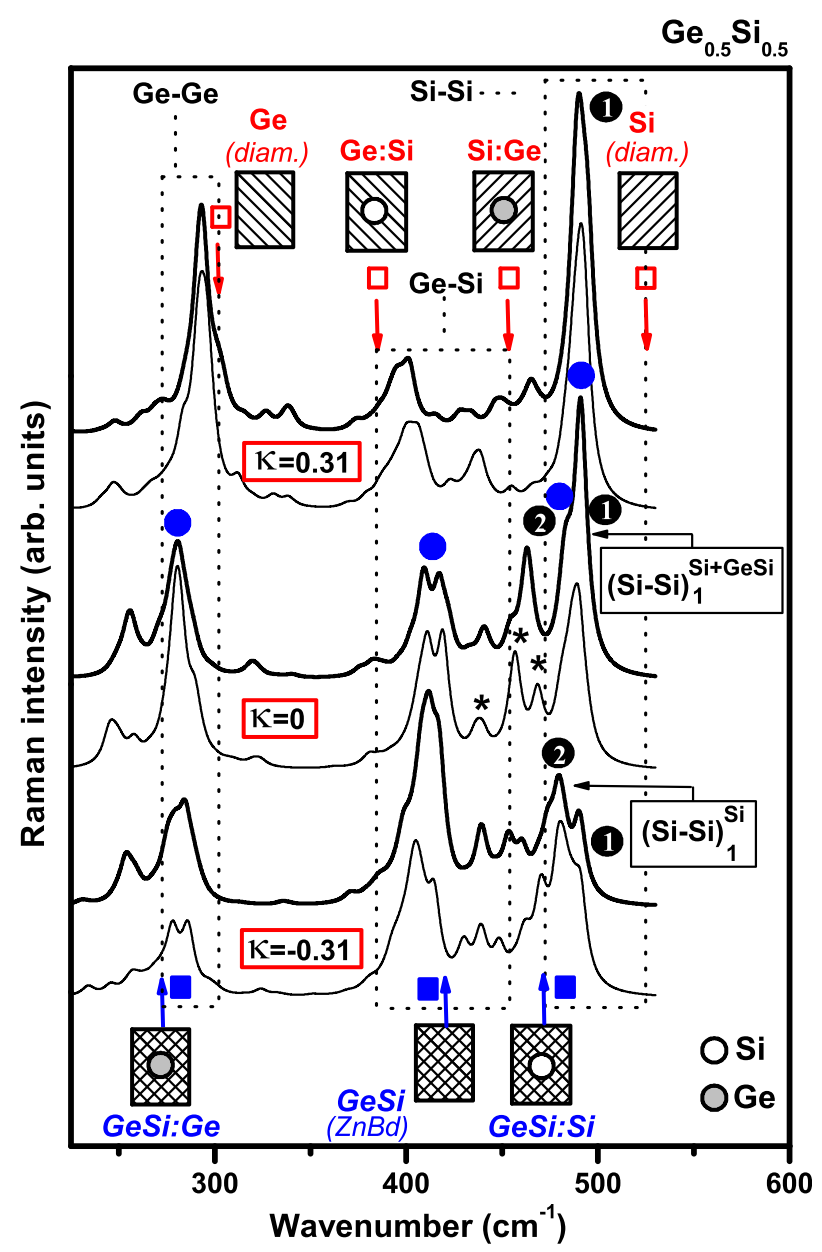

FIG. 5. Ab initio Raman spectra obtained with the three 32-atom $\mathrm{Ge}_{0.5} \mathrm{Si}_{0.5}$ supercells displayed in Fig. 4 (thick curves), corresponding to local clustering $(\kappa=+0.31$, top position), random substitution $(\kappa=0$, medium position), and local anticlustering ( $\kappa=-0.31$, bottom position). Additional $a b$ initio Raman spectra obtained by inverting the positions of the $\mathrm{Si}$ and $\mathrm{Ge}$ atoms in each supercell (thin curves), thereby leaving the $\kappa$ values unchanged, are shifted beneath the original curves, for comparison and identification of intrinsic trends. The ab initio frequencies of the unique $\mathrm{Ge}-\mathrm{Ge}$, main $\mathrm{Ge}-\mathrm{Si}$, and $\mathrm{Si}-\mathrm{Si}$ doublet in random- $\mathrm{Ge}_{0.5} \mathrm{Si}_{0.5}$ are pointed out (plainblue circles), for reference purpose. The Ge-Ge, Ge-Si, and $\mathrm{Si}-\mathrm{Si}$ spectral ranges, delimited by dotted rectangles for help in the discussion, are identified based on limit $a b$ initio frequencies when approaching full clustering (top-red arrows, open-red squares) and full anticlustering (bottom-blue arrows, plain-blue squares). Globally, the same schematic code and labeling of such limit Raman frequencies is used as in Fig. 1. The sensitive Si-Si doublet is emphasized by using numbers $(1,2)$, for unambiguous comparison with the corresponding percolation-type Raman features in Fig. 3.

feature is just absent $(\kappa \sim+0.3)$, one in which the intensity ratio between the lower and upper features reaches one third $(\kappa=0)$, and one in which the latter intensity ratio is inverted $(\kappa \sim-0.3)$. This is ideal to test the percolation scheme from $a b$ initio calculations. In principle, the refined percolation trend related to the fine structure of the $\mathrm{Si}-\mathrm{Si}$ doublet, as indicated above, should come on top of the basic MREI-like trend reflecting the relative abundance of the three bond species in the crystal when $\kappa$ varies. As already mentioned, in case of clustering this corresponds to reinforcement of the Raman signals due to the "homo" Ge-Ge and Si-Si bonds at the cost of the Raman signal due to the hetero Ge-Si bond, and vice versa in case of anticlustering.
The $a b$ initio Raman spectra obtained with the $\kappa=(+0.31,0,-0.31)$ supercells shown in Fig. 4 are displayed in (top, medium, bottom) positions of Fig. 5 (thick curves), correspondingly. A line broadening of $4 \mathrm{~cm}^{-1}$ is taken, i.e., large enough to be realistic, but small enough to allow the resolution of neighboring percolation features (the minimum splitting between neighboring percolation features is $\sim 10 \mathrm{~cm}^{-1}$, see Fig. 1). An additional series of Raman spectra obtained by inverting the $\mathrm{Si}$ and $\mathrm{Ge}$ atoms in each supercell, thereby leaving the $\kappa$ value unchanged, is slightly shifted beneath the original series, for comparison (thin curves). The two series are useful to distinguish intrinsic trends, i.e., visible in the two ab initio Raman spectra per $\kappa$ value, that deserve attention, from merely fortuitous ones, i.e., due to a particular arrangement of the $\mathrm{Si}$ and $\mathrm{Ge}$ atoms, that should not be discussed.

\section{A. Basic assignment via limit ab initio frequencies}

A prerequisite before proper comparison of the ab initio Raman spectra and of the corresponding percolation-type Raman lineshapes, as calculated within a supercell approach (3D) and along the LCA (1D), respectively, is to bridge the 3D-1D gap in the discussion of the $a b$ initio data. This comes to assign each ab initio Raman line in terms of a given bond-stretching. In principle, this can be done by examining the individual $a b$ initio vibration pattern behind each Raman line. However, in practice, this is not feasible, due to the complexity of the lattice relaxation in our disordered $\mathrm{Ge}_{0.5} \mathrm{Si}_{0.5}$ supercells, which distorts and finally obscures the atom displacements. Based on our experience, ${ }^{3,5,6,10,11}$ such direct assignment is possible only when using pure supercells, or very basic impurity motifs diluted in an otherwise pure host matrix.

We thus proceed along the latter line and delimit the Ge$\mathrm{Ge}, \mathrm{Si}-\mathrm{Si}$ and $\mathrm{Ge}-\mathrm{Si}$ spectral ranges in the ab initio Raman spectra by using a reduced set of Raman frequencies obtained by considering ideally simple impurity arrangements taken as representative of the corresponding limit bond-stretchings when approaching full c/ac. In case of clustering, such limits frequencies are accessed by using pure Ge (oblique-right hatching, see Fig. 5) and Si (oblique-left hatching) supercells, for the "homo" Ge-Ge and Si-Si bonds (1 mode per bond), and isolated $\mathrm{Ge}$ (grey symbol) or $\mathrm{Si}$ (white symbol) impurities in otherwise pure $\mathrm{Si}$ or Ge supercells, respectively, for the "hetero" Ge-Si bond ( 2 modes in total). The corresponding modes, denoted "Ge" (y=1; $\left.\kappa=+1 ; 304 \mathrm{~cm}^{-1}\right)$, "Si" (y=0; $\left.\kappa=+1 ; 522 \mathrm{~cm}^{-1}\right)$, "Si:Ge" $\left(\mathrm{y} \sim 0 ; \kappa \sim+1 ; 451 \mathrm{~cm}^{-1}\right)$, and "Ge:Si" (y $\left.\sim 1 ; \kappa \sim+1 ; 385 \mathrm{~cm}^{-1}\right)$, respectively, as observable also in Fig. 1, are marked by arrows/open-red squares at the top of Fig. 5. In case of full anticlustering, the starting supercell is a zincblende GeSi one (crossed hatching), formed with two intercalated $\mathrm{Ge}$ and $\mathrm{Si} f c c$ sublattices, thus, providing a natural insight into the "pure Ge-Si" frequency. The alternative $\mathrm{Ge}-\mathrm{Ge}$ and $\mathrm{Si}-\mathrm{Si}$ impurity frequencies are accessed by realizing a unique $\mathrm{Ge} \leftrightarrow \mathrm{Si}$ substitution either on the Si sublattice or on the Ge one of such GeSi zincblende supercell, respectively. The corresponding modes, denoted as "GeSi" $\left(\mathrm{y}=0.5 ; \kappa=-1 ; \sim 421 \mathrm{~cm}^{-1}\right)$ for the pure zincblende GeSi 
crystal, and "GeSi:Ge" $\left(\mathrm{y} \sim 0.5 ; \kappa \sim-1 ; \sim 272 \mathrm{~cm}^{-1}\right)$ or "GeSi:Si" (y $\sim 0.5 ; \kappa \sim-1 ; \sim 473 \mathrm{~cm}^{-1}$ ), for the Ge and Si impurity modes in zincblende $\mathrm{GeSi}$, respectively, are marked by arrows/plain-blue squares at the bottom of Fig. 5. They are correspondingly indicated in Fig. 1 (refer to the plain-blue squares).

Most of the above-quoted $a b$ initio frequencies were already identified in our previous work on $\mathrm{GeSi}^{6}{ }^{6}$ except the "GeSi:Ge" and "GeSi:Si" frequencies which have required a novel $a b$ initio insight in this work. The isolated $\mathrm{Ge}$ and $\mathrm{Si}$ atoms on the foreign Si-like and Ge-like fcc sublattices of the zincblende GeSi supercell generate distinct impurity modes on each side of the main Raman mode due to the host crystal. Due to the $T_{d}$ symmetry of the site occupied by such impurities, we expect triply degenerated impurity modes. ${ }^{42}$ However, this is not so in practice when using 32-atom supercells. Due to the moderate size of the 32-atom supercells, each isolated impurity in one supercell "feels" the influence of the next impurities in the surrounding supercells. The influence is not same depending on whether the next impurities in question belong to the plane formed by the short axes of the reference tetragonal supercell, or are situated along its long axis (see Fig. 4). Altogether this produces a lowering of the impurity-site symmetry, leading to the emergence of a distinct doublet per impurity (corresponding to a significant frequency gap of $\sim 10 \mathrm{~cm}^{-1}$, not shown), instead of the expected singlet. Such drawback was overcome by using larger 64-atom supercells, as already done for a similar purpose in our previous GeSi work (Ref. 6). In this case, as expected, each impurity provides a unique Raman line, located at the above-quoted "GeSi:Ge" or "GeSi:Si" frequency. The corresponding Raman spectra, obtained by using a $6 \times 6 \times 6 k$-point mesh and a typical line broadening of $4 \mathrm{~cm}^{-1}$, are shown in Fig. 6 .

We can be more explicit and assign the limit $(\mathrm{y} \sim 0,1)$ $a b$ initio Raman modes within the $[1 \times(\mathrm{Ge}-\mathrm{Ge}), 3 \times(\mathrm{Ge}-\mathrm{Si})$, $2 \times(\mathrm{Si}-\mathrm{Si})]$ percolation scheme. "Ge" and "GeSi:Ge" both refer to the same unique (Ge-Ge) percolation branch, that covers all possible Ge-Ge stretching modes in the GeSi alloy. "Ge:Si," "Si:Ge," and "GeSi" refer to Ge-Si stretching in a pure Ge environment, in a pure $\mathrm{Si}$ one and in a (Ge,Si)mixed one, respectively, thus, identifying with the side $(G e-S i)_{2}^{G e}$ and $(G e-S i)_{2}^{S i}$ percolation branches and with the intermediary $(G e-S i)_{2}^{G e S i}$ one, respectively. As for "GeSi:Si" and "Si" they are due to Si-Si stretching in a pureGe environment and in a pure-Si one, respectively, thus, assimilating with $(S i-S i)_{1}^{G e}$ and $(S i-S i)_{1}^{S i+G e S i}$, respectively. In summary, our reduced set of seven $a b$ initio frequencies covers the whole set of percolation oscillators, none is left by. The Ge-Ge, Ge-Si, and $\mathrm{Si}-\mathrm{Si}$ spectral ranges are accordingly delimited using dotted rectangles in Fig. 5.

\section{B. Raman-intensity aspect}

Now, we discuss the intensities of the as-identified $a b$ initio Raman signals in their $\kappa$-dependence. The general trend is that the Raman signals due to the "homo" Ge-Ge and $\mathrm{Si}-\mathrm{Si}$ bonds progressively reinforce with clustering, at the cost of the intermediary Raman signal due to the "hetero" Ge-Si bond, as expected. This is consistent both with the available

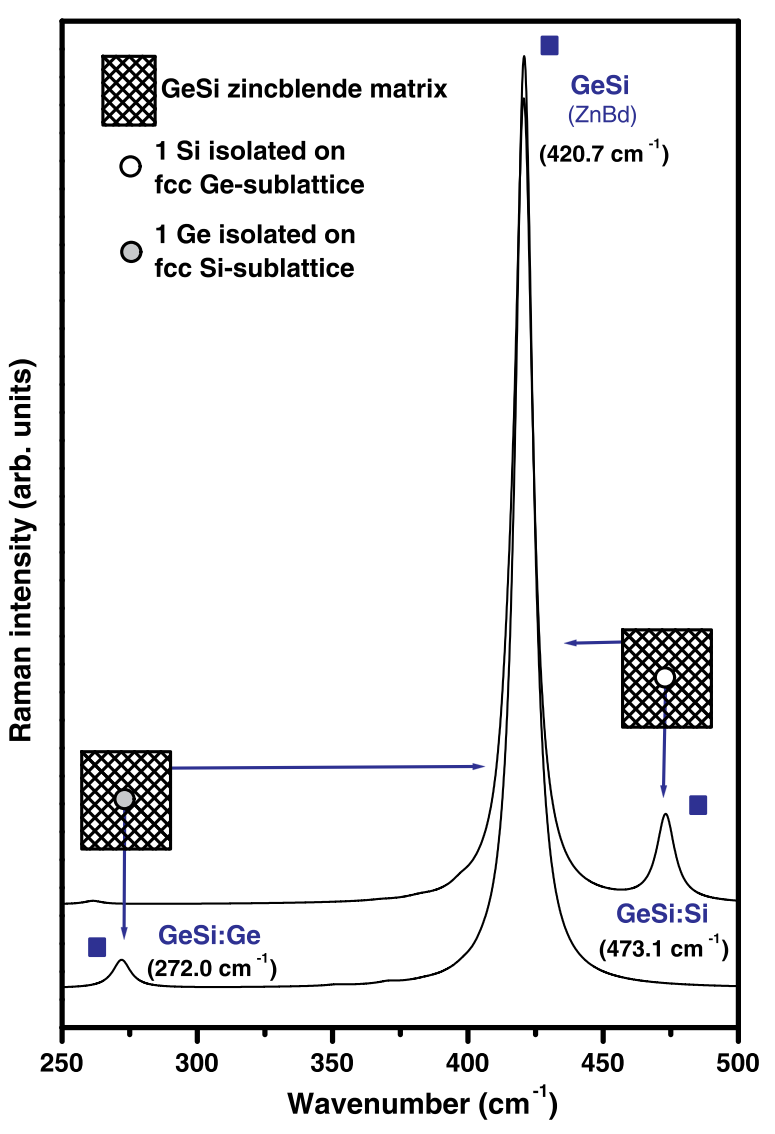

FIG. 6. Ab initio Raman spectra obtained with two 64-atom zincblende GeSi supercells containing either one isolated Si impurity on the $f c c$ Ge sublattice (top spectrum) or one isolated Ge impurity on the $f c c$ Si sublattice (bottom spectrum), as schematically indicated. Distinct modes due to the GeSizincblende host matrix and to the isolated $\mathrm{Ge}$ and $\mathrm{Si}$ impurities are labeled using the same symbol/color code as in Figs. 1 and 5, for a direct correspondence.

experimental data, in reference to point (i) (see Sec. II), and also with the predictions of the MREI and percolation schemes (see Fig. 3). We want to be more accurate and examine further the $\kappa$-dependence of the $\mathrm{Si}$-Si and $\mathrm{Ge}-\mathrm{Si}$ ab initio fine structures (being clear that the MREI scheme falls short of explaining any fine structure related to any bond).

The $a b$ initio insight is no so helpful with respect to the Ge-Si signal, in that no one-to-one correspondence can be established between the Si-Ge percolation triplet and the corresponding $a b$ initio Raman pattern. In particular, the minor/ upper component of the Ge-Si percolation triplet ideally represented by a unique feature in Fig. 3, appears to decompose into a myriad of $a b$ initio lines already in the random alloy $(\kappa=0)$-refer to the stars in Fig. 5. Such shortcoming of the GeSi percolation scheme far-off the $\mathrm{Si}$-rich limit has already been pointed out in Sec. I and also in Ref. 6. A promising trend, nevertheless, is that, in comparison with the main/ central Ge-Si feature, such extended fine structure is less represented at $\kappa=-0.31$, in spite of the large number of $\mathrm{Ge}-\mathrm{Si}$ bonds, than at $\kappa=+0.31$, in spite of the small number of Ge-Si bonds. This seems to indicate a relative reinforcement with clustering. A similar trend may be inferred for the minor/lower component of the Ge-Si triplet from the pronounced asymmetry of the main/central Ge-Si mode on its low-energy side at $\kappa=+0.31$. Altogether, this is consistent 
with the percolation scenario (see Fig. 3 and Sec. III B), and also with the existing experimental data, in reference to point (ii) (see Sec. II). However, further $a b$ initio calculations, involving much larger disordered $\mathrm{GeSi}$ supercells, in hope that the enlarged statistics on the Ge-Si bonds leads to a simplification of the extended Ge-Si fine structure (marked by stars in Fig. 5) into well-defined-possibly broadenedRaman features, are definitely needed in view to develop a meaningful comparison with the percolation triplet. Such extended ab initio calculations fall beyond the scope of this work. Our present policy with respect to the Ge-Si signal was rather to rely directly on experimental data, as detailed in Sec. III C.

The $a b$ initio insight is much more fruitful regarding the $\mathrm{Si}$-Si fine structure, our main concern in this section. The $a b$ initio Si-Si signal at $\kappa=0$ decomposes into a distinct lowerminor/upper-dominant doublet separated by $\sim 10 \mathrm{~cm}^{-1}$ (refer to the modes numbered 1 and 2 in Fig. 5) which strongly resembles its percolation analogue (refer to the central curve in Fig. 3). Moreover, only the upper mode survives at $\kappa=+0.31$, while the lower mode has turned dominant at $\kappa=-0.31$. Altogether, this is consistent with the available experimental data, in reference to (iii) (see Sec. II) Most of all, this conforms ideally to the percolation scenario, as apparent in Fig. 3. This is just the kind of independent $a b$ initio support we needed, i.e., as concerned with the impact of local c/ac at the ultimate scale of the fine structure of the Raman signal due to a given bond, i.e., $\mathrm{Si}-\mathrm{Si}$ in this case, to validate our generalized $\kappa$-dependent version of the $\mathrm{GeSi}$ percolation scheme. Additional ab initio calculations involving larger supercells, when possible, would be useful to refine the present trends by incorporating the whole statistics of the alloy disorder.

\section{Raman-frequency aspect}

Last, we cannot escape a brief discussion of the $\kappa$ dependence of the $\mathrm{Ge}_{0.5} \mathrm{Si}_{0.5}$ Raman frequencies in the $a b$ initio spectra, though this is not of direct use in this work centered on the Raman intensities. The effects of clustering $(\kappa=+0.31)$ and anticlustering $(\kappa=-0.31)$ are separately considered below, in reference to the random case $(\kappa=0)$. Accordingly, the $a b$ initio frequencies of the unique $\mathrm{Ge}-\mathrm{Ge}$, main $\mathrm{Ge}-\mathrm{Si}$ and $\mathrm{Si}-\mathrm{Si}$ doublet in the latter case are emphasized in Fig. 5 (refer to the plain-blue circles at $\kappa=0$ ), and in Fig. 1 as well. Generally, in the discussion below our attention is focused on the $\kappa$-dependence of the dominant feature in each spectral range (see Fig. 3), which fix the trend.

With clustering $(\kappa>0)$, the unique Ge-Ge mode massively blue-shifts. In contrast, the main/central Ge-Si mode undergoes a clear red-shift. As for the upper/dominant Si-Si mode, only a slight blue-shift can be detected. Such ab initio shifts, are globally consistent in nature, if not always in magnitude, with the existing experimental data in the literature. Unambiguous trends concerning the first two modes were evidenced by Schorer et al. in Ref. 18 (see Fig. 3 therein) in their careful step-by-step Raman study of an as-grown $\mathrm{Ge}_{8} \mathrm{Si}_{8}$ superlattice submitted to progressive annealing until complete destruction of the long range order, i.e., achievement of a random $\mathrm{Ge} \leftrightarrow \mathrm{Si}$ substitution. However, such study is not so helpful with respect to the remaining $\mathrm{Si}-\mathrm{Si}$ signal. This is because the $\mathrm{Si}-\mathrm{Si}$ signal develops into a proper alloy-related mode only in the very final stage of annealing, corresponding, in fact, to the last-recorded Raman spectrum of Schorer et al. Nevertheless we can appreciate in the alternative data of Schorer et al. reported together with those of Tsang et al. in Fig. 2, that clustering induces a slight blue-shift of the upper/dominant $\mathrm{Si}$-Si mode (refer to the double-horizontal arrow in Fig. 2(b)).

Such $a b$ initio shifts of the unique Ge-Ge (blue-shift, large), main/central Ge-Si (red-shift, large) and upper/dominant $\mathrm{Si}$-Si (blue-shift, small) modes find a natural explanation within the percolation scheme. Referring to the frequency-map reported in Fig. 1, it is just a matter to progress along the corresponding percolation branches from the random alloy $(\mathrm{y}=0.5, \kappa=0$, plain-blue circles) towards the relevant pure-Si or/and pure-Ge crystal(s) - the final products in case of full clustering ( $\mathrm{y}=0$ and $1, \kappa=+1$, open-red squares), as indicated by the straight (Ge-Ge, upper $\mathrm{Si}-\mathrm{Si}$ and side $\mathrm{Ge}-\mathrm{Si}$ modes) or curved (main $\mathrm{Ge}-\mathrm{Si}$ mode) red-arrows. For example, with clustering the Ge-Ge bonds tend to vibrate more in their own Ge-like environment, thus, ultimately converging to the vibration of the pure Ge crystal $(y=1 ; \kappa=+1$, open-red square). Similarly, the upper-dominant $\mathrm{Si}-\mathrm{Si}$ mode, due to Si-Si vibration in its own Si-like environment, should ultimately look like the vibration of the pure $\mathrm{Si}$ crystal $(\mathrm{y}=0 ; \kappa=+1$, open-red square). As for the main $\mathrm{Ge}-\mathrm{Si}$ mode, due to $\mathrm{Ge}-\mathrm{Si}$ stretching in a $(\mathrm{Ge}, \mathrm{Si})$-mixed environment, this may ultimately arise as well from the Si-like or Ge-like parts of the phase-separated alloy, as an impurity mode in both cases then. Accordingly, the progression is double, as reflected by antagonist curved arrows simultaneously converging onto the vibration frequencies of $\mathrm{Si}$ or $\mathrm{Ge}$ pairs of impurities in next-nearest neighbor positions (the basic motif corresponding to $\mathrm{Ge}-\mathrm{Si}$ stretching in a mixed environment as extensively explained in Ref. 6) immersed in otherwise pure $\mathrm{Ge}(\mathrm{y} \sim 1 ; \kappa=+1$, not shown) or $\mathrm{Si}(\mathrm{y} \sim 0$; $\kappa=+1$, not shown) crystals, respectively. ${ }^{6}$ Eventually, the two progressions add to generate an overall $\mathrm{Ge}-\mathrm{Si}$ red-shift. Note that the central Ge-Si mode collapses for $\kappa \geq 0.5$ (see Fig. 3), being relayed by its lower and upper satellites. These refer to Ge-Si stretching in pure-Ge and pure-Si environments, respectively, ultimately assimilating with the Ge:Si (y $\sim 1 ; \kappa=+1$, open-red square) and Si:Ge $(\mathrm{y} \sim 0 ; \kappa=+1$, open-red square) impurity modes, as indicated by the opposite dotted red-arrows (plain red-arrows are kept for the dominant modes in the considered ab initio $\kappa$-range in Fig. 5, for more clarity).

With anticlustering $(\kappa<0)$, we observe in Fig. 5 that the $a b$ initio frequencies of all dominant modes remain quasi invariant. The reference in this case is the pure GeSi zincblende crystal $(\mathrm{y}=0.5 ; \kappa=-1)$, i.e., the ultimate host crystal in case of full anticlustering. The phonon shifts are thus discussed from Fig. 1 by using curved (semi-closed loops) bluearrows starting from frequencies identified on the relevant percolation branches of the random GeSi alloy $(y=0.5$; $\kappa=0$, plain-blue dots in Figs. 1 and 5) and ending at the corresponding frequencies in zincblende $\mathrm{GeSi}(\mathrm{y}=0.5 ; \kappa \sim-1$, 
plain-blue squares in Figs. 1 and 5). For example, the central/main Ge-Si mode, due to $\mathrm{Ge}-\mathrm{Si}$ vibration in a $(\mathrm{Ge}, \mathrm{Si})$ mixed environment naturally converges onto the frequency of the pure zincblende $\mathrm{GeSi}(\mathrm{y}=0.5 ; \kappa=-1$, plain-blue dot, centre of Fig. 1). As for the unique Ge-Ge mode and lower/ main $\mathrm{Si}-\mathrm{Si}$ component of the $\mathrm{Si}-\mathrm{Si}$ doublet, these assimilate in fine with the $\mathrm{GeSi}: \mathrm{Ge}(\mathrm{y}=0.5 ; \kappa \sim-1$, plain-blue dot, bottom of Fig. 1) and GeSi:Si $(y=0.5 ; \kappa \sim-1$, plain-blue dot, top of Fig. 1) impurity modes. We recall that the latter correspond to a unique $\mathrm{Ge} \leftrightarrow \mathrm{Si}$ substitution on the $f c c \mathrm{Si}$ or $\mathrm{Ge}$ sub-lattices of zincblende $\mathrm{GeSi}$, respectively. In each case, the starting and ending frequencies differ by less than a few centimeters inverse, corresponding in practice to quasi invariance of the Raman frequencies.

\section{CONCLUSION}

We report on a comprehensive study of the intensities of the Raman multi-lines of the diamond-type GeSi alloy in their (co-)dependence on local clustering/anticlustering within the percolation scheme, which has recently lead to a novel understanding of the random-GeSi Raman pattern along the linear chain approximation in terms of six basic 1D oscillators $[1 \times(\mathrm{Ge}-\mathrm{Ge}), 3 \times(\mathrm{Ge}-\mathrm{Si}), 2 \times(\mathrm{Si}-\mathrm{Si})] .{ }^{6}$ For doing so, the individual fractions of such $1 \mathrm{D}$-oscillators are expressed via a relevant order parameter $\kappa$. This is introduced along the approach used by Verleur and Barker in their 1D cluster model that distinguishes between all possible first neighbor oscillators of a 3D zincblende alloy. ${ }^{8}$ However, care is taken to overcome some ambiguity behind the 1D-3D ambivalence in the treatment of Verleur and Barker by adopting a pure 1D formalism in the present work. Moreover, the formalism is generalized to the GeSi-like diamond structure. Also, not only the first-neighbor environments of a bond are covered, but also the second-neighbor ones, in reference to the $\mathrm{Si}-\mathrm{Si}$ doublet and to the Ge-Si triplet, respectively. Last, the GeSi-diamond version takes into account inter-bond transfer of oscillator strength on top of the zincblende-like intra-bond transfer of oscillator strength driven by local clustering/anticlustering. Predictive $\kappa$-dependent $\mathrm{Ge}_{0.5} \mathrm{Si}_{0.5}$ percolation Raman lineshapes compare fairly well with (i) existing experimental data, when referring to the Ge-Si fine structure, and with (ii) ab initio Raman spectra calculated by using (32-atom) disordered supercells matching the required $\kappa$ values, referring then to the $\mathrm{Si}-\mathrm{Si}$ fine structure.

Altogether, the present work formalizes an intrinsic ability behind Raman scattering to achieve a quantitative insight into clustering/anticlustering in an alloy at the very local scale. This was still lacking so far. Interestingly, such formalization is achieved along the linear chain approximation, i.e., simply at $1 \mathrm{D}$.

\section{ACKNOWLEDGMENTS}

We are grateful to A. V. Postnikov for a critical reading of the manuscript and many useful discussions, and also to M. I. Alonso for stimulating our interest on clustering/ anticlustering issues in GeSi. This work was supported by the European funding of Region Lorraine under Project FEDER-Percalloy No. presage 34619. V.J.B.T. would like to thank for funding by the Fundação para a Ciência e a Tecnologia, Portugal (FCT) under the Grant PEst-C/CTM/ LA0025/2011.

${ }^{1}$ A. Mascarenhas, Spontaneous Ordering in Semiconductor Alloys, edited by (Kluwer Academics, New York, 2002).

${ }^{2}$ In a crude description, the phonon frequency square scales as the effective restoring force constant of a bond divided by its reduced mass.

${ }^{3}$ A. V. Postnikov, O. Pagès, and J. Hugel, Phys. Rev. B 71, 115206 (2005).

${ }^{4}$ T. Ganguli, J. Mazher, A. Polian, S. K. Deb, F. Villain, O. Pagès, W. Paszkowicz, and F. Firszt, J. Appl. Phys. 108, 083539 (2010).

${ }^{5}$ O. Pagès, J. Souhabi, A. V. Postnikov, and A. Chafi, Phys. Rev. B 80, 035204 (2009).

${ }^{6}$ O. Pagès, J. Souhabi, V. J. B. Torres, A. V. Postnikov, and K. C. Rustagi, Phys. Rev. B 86, 045201 (2012).

${ }^{7}$ I. F. Chang and S. S. Mitra, Adv. Phys. 20, 359 (1971).

${ }^{8}$ H. W. Verleur and A. S. Barker, Phys. Rev. 149, 715 (1966).

${ }^{9}$ B. V. Robouch, A. Kisiel, and E. M. Sheregii, Phys. Rev. B 64, 073204 (2001).

${ }^{10}$ O. Pagès, A. V. Postnikov, M. Kassem, A. Chafi, A. Nassour, and S. Doyen, Phys. Rev. B 77, 125208 (2008).

${ }^{11}$ O. Pagès, A. V. Postnikov, A. Chafi, D. Bormann, P. Simon, F. Glas, F. Firszt, W. Paszkowicz, and E. Tournié, Eur. Phys. J. B 73, 461 (2010).

${ }^{12}$ S. Kanakaraju, A. K. Sood, and S. Mohan, Phys. Rev. B 61, 8334 (2000).

${ }^{13}$ N. Nakano, L. Marville, S.-M. Jang, K. Liao, C. Tsai, J. Tsai, H.-W. Kim, and R. Reif, J. Appl. Phys. 73, 414 (1993).

${ }^{14}$ J. S. Reparaz, A. Bernardi, A. R. Goñi, M. I. Alonso, and M. Garriga, Appl. Phys. Lett. 92, 081909 (2008).

${ }^{15}$ J. C. Tsang, V. P. Kesan, J. L. Freeouf, F. K. LeGoues, and S. S. Iyer, Phys. Rev. B 46, 6907 (1992).

${ }^{16}$ F. Cerdeira, M. I. Alonso, D. Niles, M. Garriga, M. Cardona, E. Kasper, and H. Kibbel, Phys. Rev. B 40, 1361 (1989).

${ }^{17}$ C. Sheng, T.-C. Zhou, Q. Cai, D. Gong, M.-R. Yu, X.-J. Zhang, and X. Wang, Phys. Rev. B 53, 10771 (1996).

${ }^{18}$ R. Schorer, E. Friess, K. Eberl, and G. Abstreiter, Phys. Rev. B 44, 1772 (1991).

${ }^{19}$ R. Schorer, G. Abstreiter, H. Kibbel, and H. Presting, Phys. Rev. B 50, 18211 (1994).

${ }^{20}$ M. I. Alonso, F. Cerdeira, D. Niles, M. Cardona, E. Kasper, and H. Kibbel, J. Appl. Phys. 66, 5645 (1989).

${ }^{21}$ S. de Gironcoli, E. Molinari, R. Schorer, and G. Abstreiter, Phys. Rev. B 48, 8959 (1993).

${ }^{22}$ R. Schorer, G. Abstreiter, S. de Gironcoli, E. Molinari, H. Kibbel, and H. Presting, Phys. Rev. B 49, 5406 (1994).

${ }^{23}$ M. I. Alonso and K. Winer, Phys. Rev. B 39, 10056 (1989).

${ }^{24}$ J. Zi, K. Zhang, and X. Xie, Phys. Rev. B 47, 9937 (1993).

${ }^{25}$ J. Zi, W. Ludwig, K. Zhang, and X. Xie, Phys. Rev. B 51, 7886 (1995).

${ }^{26}$ J. S. Reparaz, I. C. Marcus, A. R. Goñi, M. Garriga, and M. I. Alonso, J. Appl. Phys. 112, 023512 (2012).

${ }^{27}$ Note that the GeSi zincblende crystal can be described in terms of alternate $\mathrm{Si}$ and $\mathrm{Ge}$ (111) planes into a [111]-oriented $\mathrm{Ge}_{1} \mathrm{Si}_{1}$ periodical sequence, using the standard notation of $\mathrm{a} \mathrm{Ge}_{\mathrm{n}} \mathrm{Si}_{\mathrm{m}}$ superlattice.

${ }^{28}$ P. R. Briddon and R. Jones, Phys. Status Solidi B 217, 131 (2000).

${ }^{29}$ P. J. Rayson and R. Briddon, Comput. Phys. Commun. 178, 128 (2008).

${ }^{30}$ J. P. Perdew and Y. Wang, Phys. Rev. B 45, 13244 (1992).

${ }^{31}$ C. Hartwigsen, S. Goedecker, and J. Hutter, Phys. Rev. B 58, 3641 (1998).

${ }^{32}$ J. P. Goss, M. J. Shaw, and P. R. Briddon, Top. Appl. Phys. 104, 69-94 (2007).

${ }^{33}$ V. J. B. Torres, J. Coutinho, P. R. Briddon, and M. Barroso, Thin Solid Films 517, 395 (2008).

${ }^{34}$ H. J. Monkhorst and J. D. Pack, Phys. Rev. B 13, 5188 (1976).

${ }^{35}$ P. J. Mohr and B. N. Taylor, Rev. Mod. Phys. 72, 351 (2000).

${ }^{36}$ J. Singh, Physics of Semiconductors and Their Heterostructures (McGraw-Hill, New York, 1993).

${ }^{37}$ C. R. S. da Silva, P. Venezuela, A. J. R. da Silva, and A. Fazzio, Solid State Commun. 120, 369 (2001). 
${ }^{38}$ Landbolt-Börnstein, Numerical Data and Functional Relationships in Science and Technology-Crystal and Solid State Physics (Springer, Berlin, 1984), Vol. 18.

${ }^{39}$ G. Dolling, in Proceedings of 2nd Symposium on Inelastic Scattering of Neutron in Solid and Liquids, Chalk River, Canada (1962), p. 37.
${ }^{40}$ J. H. Parker, Jr., D. W. Feldman, and M. Ashkin, Phys. Rev. 155, 712 (1967).

${ }^{41}$ H. M. Abduljalil, M. A. Abdulsattar, and S. R. Al-Mansoury, Micro Nano Lett. 6, 386 (2011).

${ }^{42}$ D. N. Talwar, Z. C. Feng, and T.-R. Yang, Phys. Rev. B 85, 195203 (2012). 